In cooperation with the Harrison County Development Commission

\title{
Quality of Water in Selected Wells, Harrison County, Mississippi, 1997-2005
}

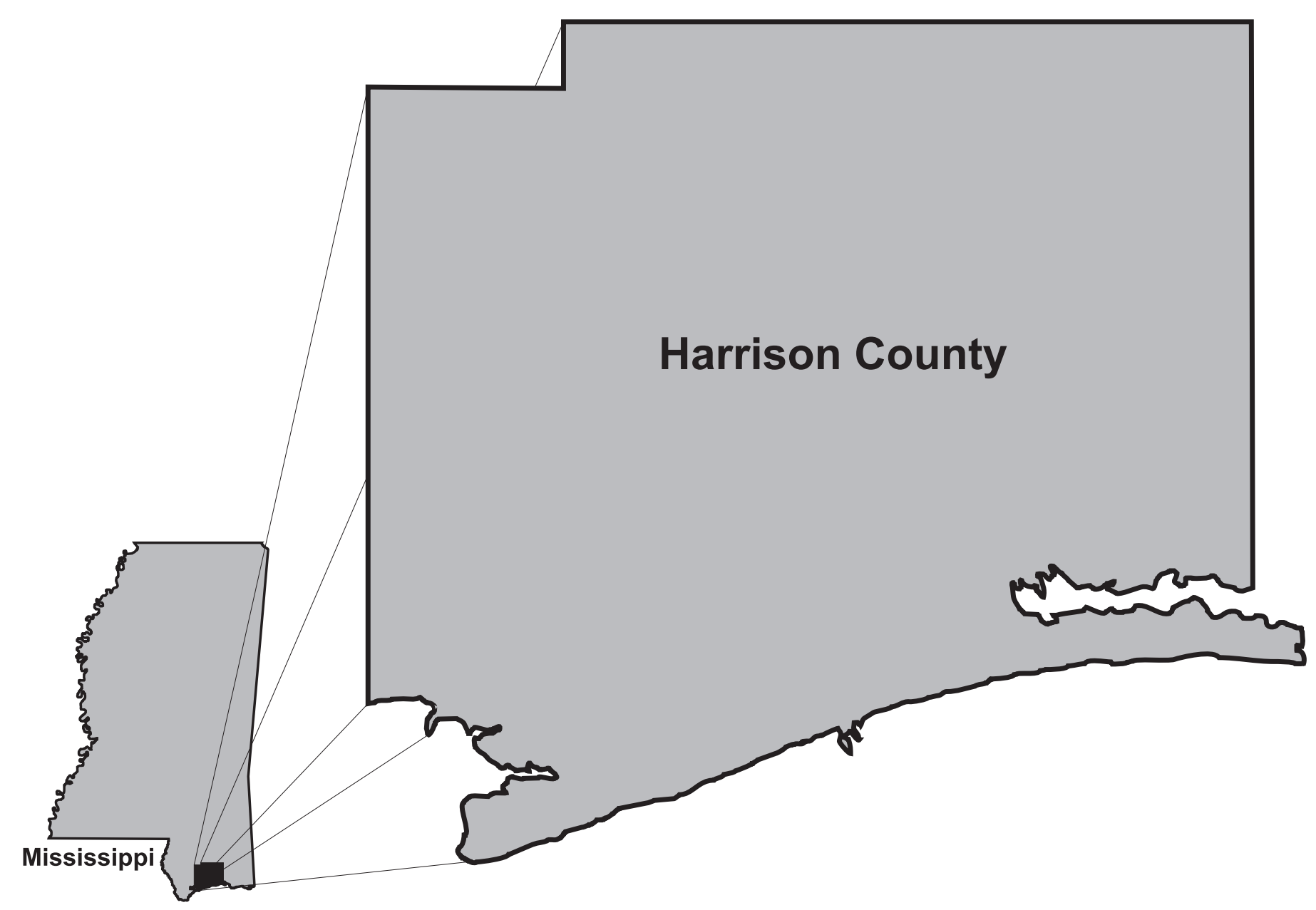

Open-File Report 2007-1287 


\section{Quality of Water in Selected Wells, Harrison County, Mississippi 1997-2005}

By David E. Burt, Jr. and Heather L. Welch

In cooperation with the Harrison County Development Commission

Open-File Report 2007-1287 


\title{
U.S. Department of the Interior DIRK KEMPTHORNE, Secretary
}

\author{
U.S. Geological Survey \\ Mark D. Myers, Director
}

\section{U.S. Geological Survey, Reston, Virginia: 2007}

For product and ordering information:

World Wide Web: http://www.usgs.gov/pubprod

Telephone: 1-888-ASK-USGS

For more information on the USGS--the Federal source for science about the Earth, its natural and living resources, natural hazards, and the environment:

World Wide Web: http://www.usgs.gov

Telephone: 1-888-ASK-USGS

Any use of trade, product, or firm names is for descriptive purposes only and does not imply endorsement by the U.S. Government.

Although this report is in the public domain, permission must be secured from the individual copyright owners to reproduce any copyrighted materials contained within this report.

Suggested citation:

Burt, Jr., D.E., and Welch, H.L., 2007, Quality of water in selected wells, Harrison County, Mississippi, 1997-2005: U.S. Geological Survey Open-File Report 2007-1287, 16 p. 


\section{Contents}

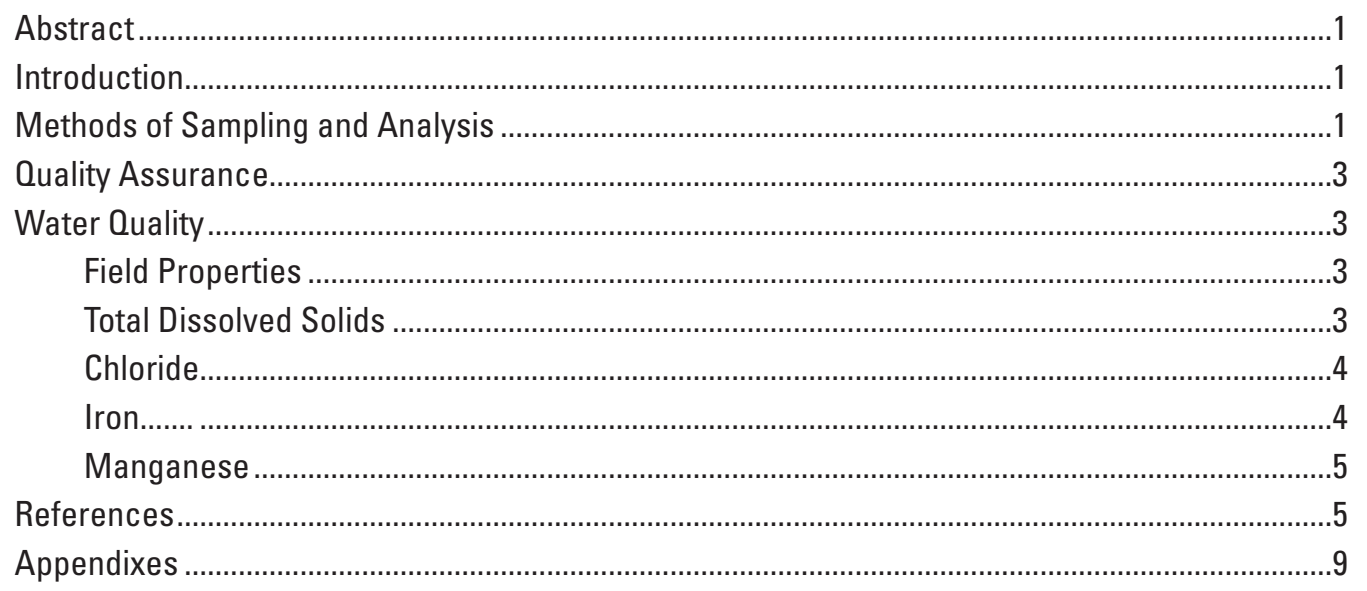




\section{Figures}

1. Map showing locations of wells sampled in Harrison County, Mississippi, 1997-2005 ...............2

2. Graph showing distribution of total dissolved solids concentrations in the Graham Ferry, Pascagoula, and Hattiesburg aquifers of Harrison County, Mississippi, 1997-2005 .........4

3. Graph showing distribution of chloride concentrations in the Graham Ferry, Pascagoula, and Hattiesburg aquifers of Harrison County, Mississippi, 1997-2005..............................4

4. Graph showing distributions of iron concentrations in the Graham Ferry, Pascagoula, and Hattiesburg aquifers of Harrison County, Mississippi, 1997-2005................................5

5. Graph showing distribution of manganese concentrations in the Graham Ferry, Pascagoula, and Hattiesburg aquifers of Harrison County, Mississippi, 1997-2005 ........5

\section{Tables}

1. Selected site information for wells sampled in Harrison County, Mississippi, 1997-2005 


\begin{tabular}{lll}
\hline \multicolumn{1}{c}{ Multiply } & \multicolumn{1}{c}{ By } & \multicolumn{1}{c}{ To obtain } \\
\hline \multicolumn{2}{c}{ Length } \\
\hline inch (in.) & 2.54 & centimeter $(\mathrm{cm})$ \\
foot (ft) & 0.3048 & meter $(\mathrm{m})$ \\
\hline \multicolumn{2}{c}{ Area } & \\
\hline square mile $\left(\mathrm{mi}^{2}\right)$ & 2.590 & square kilometer $\left(\mathrm{km}^{2}\right)$ \\
\hline
\end{tabular}

Specific conductance is given in microsiemens per centimeter at 25 degrees Celsius $(\mu \mathrm{S} / \mathrm{cm}$ at $\left.25^{\circ} \mathrm{C}\right)$.

Concentrations of chemical constituents in water are given either in milligrams per liter (mg/L) or micrograms per liter $(\mu \mathrm{g} / \mathrm{L})$.

Color is given in platinum-cobalt (Pt-Co) units. 


\title{
Quality of Water in Selected Wells, Harrison County, Mississippi, 1997-2005
}

\author{
By David E. Burt, Jr. and Heather L. Welch
}

\section{ABSTRACT}

The U.S. Geological Survey collects, on a systematic basis, data needed to determine and evaluate the ground-water resources of Harrison County, Mississippi. Water samples were collected from 1997 to 2005 at selected wells screened in the Citronelle, Graham Ferry, Pascagoula, Hattiesburg, and Catahoula Sandstone aquifers and were analyzed for field properties (temperature, $\mathrm{pH}$, specific conductivity, and color), total dissolved solids, chloride, iron, and manganese. The U.S. Environmental Protection Agency Secondary Drinking Water Regulation (SDWR) of 500 milligrams per liter for total dissolved solids was exceeded in water from three wells in the Hattiesburg aquifer and 12 wells in the Pascagoula aquifer. Chloride concentrations did not exceed the SDWR in any of the aquifers sampled, except for the Pascagoula aquifer where water in 10 wells had concentrations that exceeded the SDWR of 250 milligrams per liter. Iron concentrations in water from nine wells completed in the Graham Ferry and four wells completed in the Pascagoula aquifers exceeded the SDWR of 300 micrograms per liter. Thirty water samples collected from the Graham Ferry and 12 from the Pascagoula aquifer exceeded the SDWR of 50 micrograms per liter for manganese.

\section{INTRODUCTION}

The U.S. Geological Survey (USGS) collects, on a systematic basis, data needed to determine and evaluate the ground-water resources of Harrison County, Mississippi. Water samples are collected from the aquifers underlying Harrison County to assess water-quality characteristics and to determine changes in water quality. The resulting information forms the foundation for many of the area's water-resources management and planning activities and allows for the early detection of possible problems - for example, saltwater intrusion and contamination. Since April 1997, the USGS has collected water-quality samples from about 25 wells (of an approximately 100-well network), annually, for 4 consecutive years. Each year a different set of 25 wells was sampled. Then, during the fifth year, the original 25 wells were re-sampled, and the cycle was repeated. As much as possible, wells that were damaged, destroyed, abandoned, or otherwise no longer available for sampling were replaced in the network with wells completed in the same aquifer. Thus, the nominal 100-well network has included 104 wells.

Sites were selected to represent the major geologic units and depths at which wells have been completed in Harrison County (table 1). Two of the 104 wells were completed in the Citronelle Formation, 45 in the Graham Ferry Formation, 48 in the Pascagoula Formation, 8 in the Hattiesburg Formation, and 1 in the Catahoula Sandstone; hereafter, in this report, these formations are referred to as the Citronelle, Graham Ferry, Pascagoula, Hattiesburg, and Catahoula aquifers, respectively.

This report presents results of the analyses of water samples collected from selected wells in Harrison County from 1997 to 2005. Temperature, $\mathrm{pH}$, specific conductance, and color were monitored and recorded for all of the wells, and each well was sampled for chloride, dissolved solids, iron, and manganese. These constituents were compared to U.S. Environmental Protection Agency (USEPA) drinking-water standards for a frame of reference (U.S. Environmental Protection Agency, 2004).

\section{METHODS OF SAMPLING AND ANALYSES}

Water samples were collected (generally in April) from wells in Harrison County (fig. 1) from the Citronelle, Graham Ferry, Pascagoula, Hattiesburg, and Catahoula aquifers (in order from shallowest to deepest). The wells were pumped prior to sampling to withdraw enough water from the casing to allow the $\mathrm{pH}$, and specific conductance to stabilize, thus ensuring the sample collected would accurately represent water from the aquifer. Final field (in situ) measurements of temperature, $\mathrm{pH}$, and specific conductance were recorded. The samples were then shipped to the USGS National Water Quality Laboratory (NWQL) in Lakewood, CO, where the samples were analyzed using standard USGS procedures. Samples collected prior to 2004 were analyzed at the Water Quality Service Unit in Ocala, FL. Most samples were analyzed for color, $\mathrm{pH}$, specific conductance, and concentrations of chloride, dissolved solids, iron, and manganese (Appendix I). 


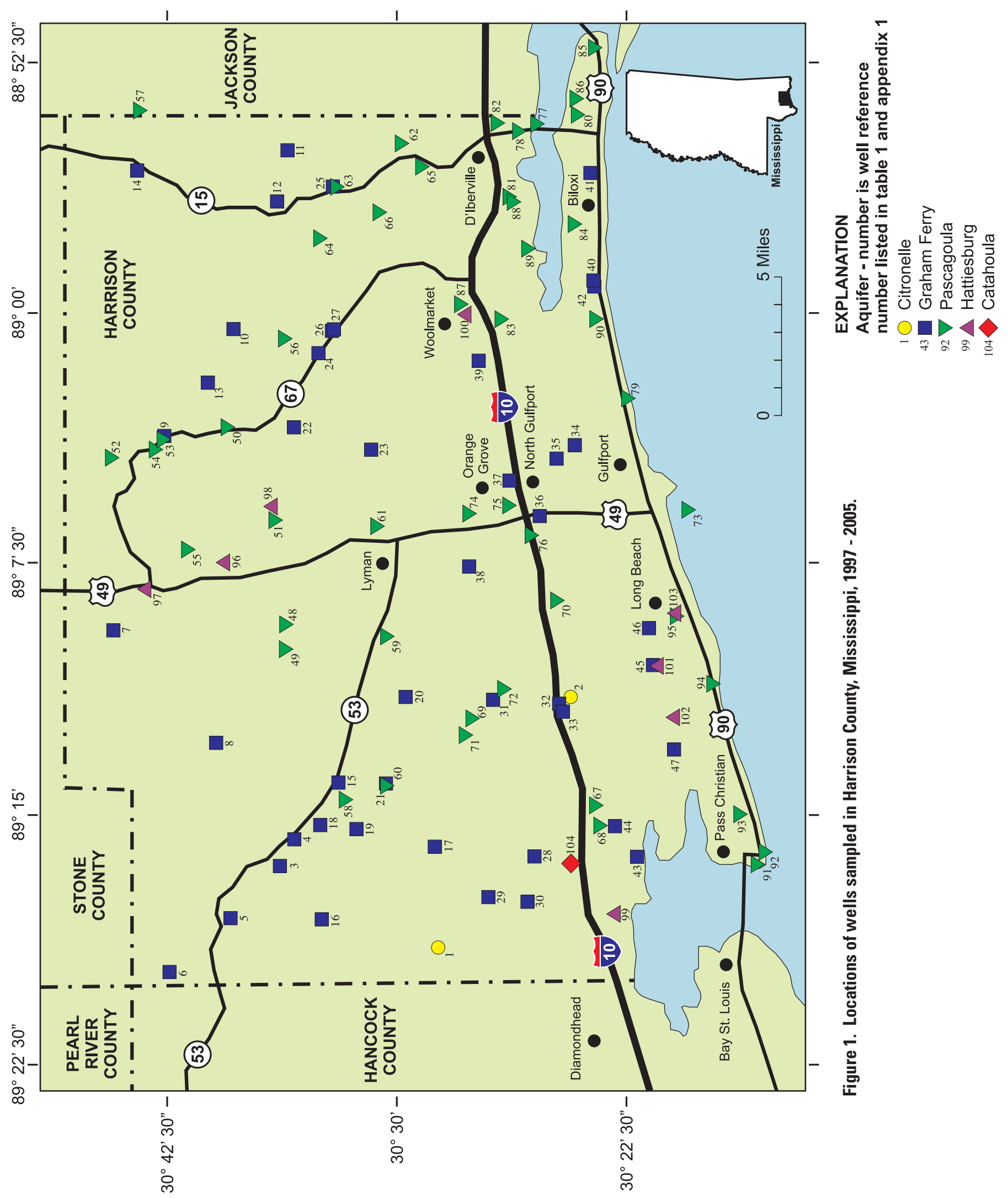




\section{QUALITY ASSURANCE}

The USGS participates annually in the National Field Quality Assurance (NFQA) Program. This program tests the accuracy of the equipment used in the field and the technician's skills at using this equipment. In addition, the USGS routinely collects quality-control samples to substantiate the validity of the data that the agency reports to its cooperators and the public. During the sampling period in April 2005, two replicate samples and one blank sample were collected. The replicate samples show the amount of variability in the samples and indicate any potential problems associated with the sampling process. The blank sample is collected from water that is prepared at the NWQL, free from any analytes being sampled for, and is used to identify any problems or deficiencies in field-cleaning procedures that could bias the results. Quality-control data are listed in Appendix II.

\section{WATER OUALITY}

Concentrations of chloride, dissolved solids, iron, and manganese for each well sampled since 1997 are presented graphically with box plots which show the distribution of the water-quality data. These four constituents were chosen because of their importance to the quality of drinking water. Chloride, along with dissolved solids, affects the taste of the water. Iron and manganese in water cause undesirable brownish-colored stains on porcelain plumbing fixtures and laundry. Water with high levels of iron and manganese can also have a foul taste and a bad odor. The results are discussed, by aquifer, below. The results may not be representative of the entire areal extent of the aquifers underlying Harrison County, but are representative of the aquifers at the sampled wells. Although the continued sampling effort is designed, with time, to produce enough long-term, distributed measurements to define waterquality trends for the aquifers, additional sampling will be necessary to determine the source and significance of changes in these water-quality measurements.

For most of these water-quality properties and constituents, no systematic change was detected. However, substantial changes occurred at a small number of individual wells. Additional sampling will be necessary to determine the source and significance of these changes.

\section{Field Properties}

The two wells in the Citronelle aquifer had depths of 70 and 84 feet (table 1). A pH value of 4.8, which is lower than the USEPA SDWR range of 6.5 to 8.5 , was recorded at one well. Specific conductivity ranged from 21 to 202 microsiemens per centimeter $(\mu \mathrm{S} / \mathrm{cm})$ in four samples, with a median value of $111 \mu \mathrm{S} / \mathrm{cm}$. Color in five samples ranged from $<5$ to 5 platinum-cobalt (Pt-Co) units, with a median value of $5 \mathrm{Pt}-\mathrm{Co}$ units.
Well depths in the Graham Ferry aquifer ranged from 63 to 829 feet, with a median value of 467 feet in the 45 sampled wells (table 1). Field measurements of $\mathrm{pH}$ ranged from 5 to 9.2 in 85 samples, with a median value of 7.7. Six samples were below the SDWR of 6.5, and eight samples were above the SDWR limit of 8.5. Specific conductivity ranged from 20 to $540 \mu \mathrm{S} / \mathrm{cm}$ in 89 samples, with a median of $203 \mu \mathrm{S} / \mathrm{cm}$. Color in 90 samples ranged from $<1$ to $50 \mathrm{Pt}-\mathrm{Co}$ units, with a median value of $9 \mathrm{Pt}-\mathrm{Co}$ units.

Well depths for the 48 wells sampled in the Pascagoula aquifer ranged from 476 to 1,320 feet, with a median depth of 807.5 feet (table 1). Field measurements of $\mathrm{pH}$ ranged from 5 to 10.2 in 98 samples, with a median value of 8.6. Four values were below the SDWR of 6.5 , and 59 values exceeded the upper SDWR limit of 8.5. Specific conductivity ranged from 28 to $1,270 \mu \mathrm{S} / \mathrm{cm}$ in 101 samples, with a median of 310 $\mu \mathrm{S} / \mathrm{cm}$. Color in 94 samples ranged from $<1$ to $50 \mathrm{Pt}-\mathrm{Co}$ units, with a median value of $10 \mathrm{Pt}-\mathrm{Co}$ units.

Well depths for the eight wells sampled in the Hattiesburg aquifer ranged from 1,140 to 1,860 feet, with a median depth of 1,560 feet (table 1). Field measurements of $\mathrm{pH}$ ranged from 8.2 to 9.2 in 18 samples, with a median value of 8.8. Sixteen $\mathrm{pH}$ values exceeded the upper SDWR limit of 8.5. Specific conductivity ranged from 26 to $1,270 \mu \mathrm{S} / \mathrm{cm}$ in 20 samples, with a median of $436 \mu \mathrm{S} / \mathrm{cm}$. Color in 20 samples ranged from $<5$ to $30 \mathrm{Pt}-\mathrm{Co}$ units, with a median value of $10 \mathrm{Pt}-\mathrm{Co}$ units.

The well sampled in the Catahoula aquifer had a total depth of 2,413 feet (table 1). The two field measurements of pH were 8.7, which exceeded the upper SDWR limit of 8.5. Specific conductivities in two samples were 593 and $595 \mu \mathrm{S} /$ $\mathrm{cm}$. Color in two samples was 10 and $20 \mathrm{Pt}-\mathrm{Co}$ units.

\section{Total Dissolved Solids}

Total dissolved-solids concentrations in four samples from two wells in the Citronelle aquifer ranged from 20 to 162 milligrams per liter $(\mathrm{mg} / \mathrm{L})$, with a median value of 87 $\mathrm{mg} / \mathrm{L}$. Although the two wells had large differences in total dissolved-solids concentrations, resampling during the fifth year of the cycle produced similar results. The lowest values of total dissolved-solids concentrations generally were from wells screened in the Graham Ferry aquifer (fig. 2). Total dissolved-solids concentrations ranged from 15 to $334 \mathrm{mg} / \mathrm{L}$, with a median value of $160 \mathrm{mg} / \mathrm{L}$. The highest values of total dissolved-solids concentrations generally were from wells screened in the Pascagoula aquifer (fig. 2). Total dissolvedsolids concentrations ranged from 16 to $990 \mathrm{mg} / \mathrm{L}$, with a median value of $200 \mathrm{mg} / \mathrm{L}$. Detections in water at 12 wells were above the SDWR of $500 \mathrm{mg} / \mathrm{L}$ for total dissolved solids. Total dissolved-solids concentrations in the Hattiesburg aquifer ranged from 20 to $704 \mathrm{mg} / \mathrm{L}$, with a median value of $266 \mathrm{mg} / \mathrm{L}$ (fig. 2). Concentrations in three wells exceeded the SDWR for total dissolved-solids. Total dissolved-solids concentrations of 328 and $346 \mathrm{mg} / \mathrm{L}$ were measured in 1997 and 2001, respectively, for a single well screened in the Catahoula aquifer. Changes in total dissolved-solids concentrations over time in each aquifer were small, and no overall trend was observed. 


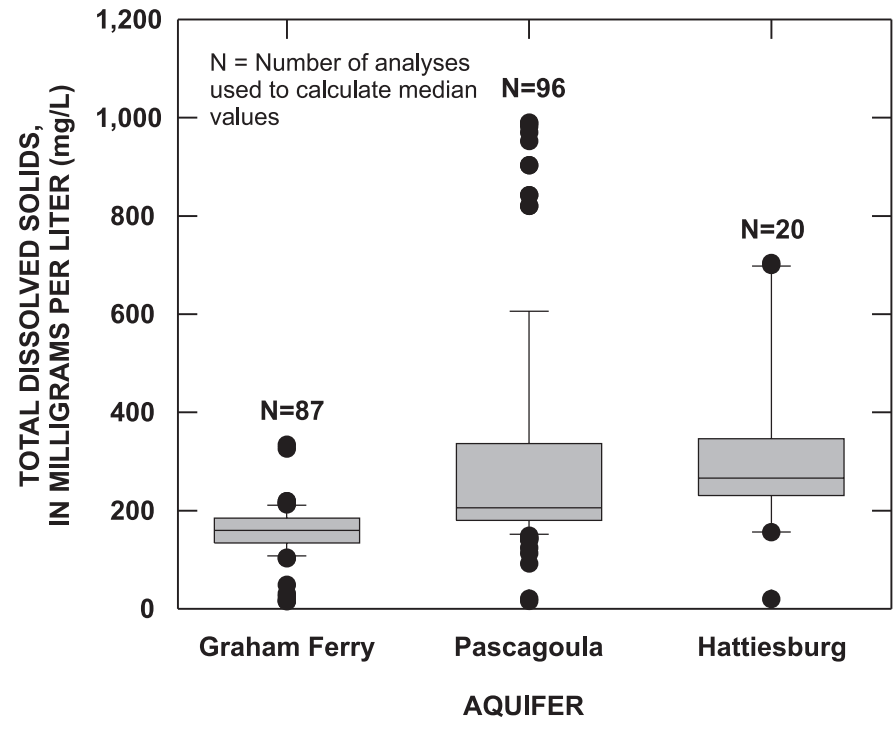

Figure 2. Distribution of total dissolved-solids concentrations in the Graham Ferry, Pascagoula, and Hattiesburg aquifers of Harrison County, Mississippi, 1997-2005.

\section{Chloride}

Chloride concentrations for the Citronelle aquifer are limited to data for two wells. Each well was sampled twice, and the concentrations were nearly identical, ranging from 3.3 to $3.4 \mathrm{mg} / \mathrm{L}$.

Chloride concentrations ranged from 2.2 to $18 \mathrm{mg} / \mathrm{L}$, with a median value of $3.25 \mathrm{mg} / \mathrm{L}$, for the wells screened in the Graham Ferry aquifer (fig. 3). All concentrations were well below the SDWR for chloride. For wells that have been sampled multiple times since 1997, the chloride concentrations have remained nearly the same from year to year, varying by $2 \mathrm{mg} / \mathrm{L}$ or less.

The highest chloride concentrations generally were from wells screened in the Pascagoula aquifer. Chloride concentrations in the Pascagoula aquifer ranged from 2.0 to $430 \mathrm{mg} / \mathrm{L}$, with a median value of $5.0 \mathrm{mg} / \mathrm{L}$ (fig. 3). Water in 10 wells exceeded the SDWR for chloride. Water-quality data since 1997 indicate that although chloride concentrations generally have remained nearly constant for most wells screened in the Pascagoula aquifer, there was a slight increase with time. Site 86 (well M0790) had the largest difference over time, an increase from $310 \mathrm{mg} / \mathrm{L}$ in 1997 to $347 \mathrm{mg} / \mathrm{L}$ in 2005.

Chloride concentrations ranged from 1.9 to $230 \mathrm{mg} / \mathrm{L}$, with a median value of $23.6 \mathrm{mg} / \mathrm{L}$, for wells screened in the Hattiesburg aquifer (fig. 3). No concentrations exceeded the SDWR for chloride. For most wells with multiple samplings, chloride concentrations varied by less than $5 \mathrm{mg} / \mathrm{L}$. The maximum change in chloride concentrations was a decrease of 11 $\mathrm{mg} / \mathrm{L}$ from 1997 to 2005 at site 100 (well M0711).

In 1997, a chloride concentration of $76 \mathrm{mg} / \mathrm{L}$ was measured in the single well screened in the Catahoula aquifer. When this well was sampled again in 2001, the chloride concentration was $75 \mathrm{mg} / \mathrm{L}$.

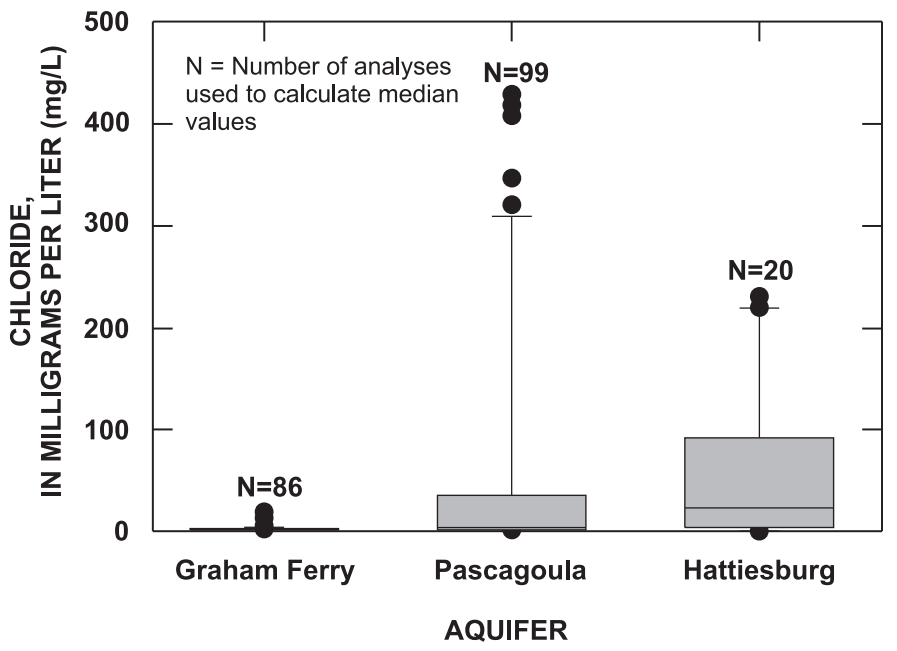

Figure 3. Distribution of chloride concentrations in the Graham Ferry, Pascagoula, and Hattiesburg aquifers of Harrison County, Mississippi, 1997-2005.

Iron

Iron concentrations in water from the Citronelle aquifer were analyzed in samples from two wells through time. Each well was sampled twice, and the iron concentrations ranged from 6 to 70 micrograms per liter $(\mu \mathrm{g} / \mathrm{L})$ - well below the SDWR of $300 \mu \mathrm{g} / \mathrm{L}$. The median value was $37 \mu \mathrm{g} / \mathrm{L}$. Differences in concentrations during resampling were small and statistically insignificant.

Iron concentrations ranged from less than 2 to 3,330 $\mu \mathrm{g} / \mathrm{L}$, with a median value of $47.5 \mu \mathrm{g} / \mathrm{L}$, for the wells screened in the Graham Ferry aquifer (fig. 4). Water in nine wells exceeded the SDWR limit for iron. For three wells that were sampled multiple times, iron concentrations increased substantially. Concentrations at site 5 (well A0049) increased from 57 to $580 \mu \mathrm{g} / \mathrm{L}$. An increase from 2,600 to 3,330 $\mu \mathrm{g} / \mathrm{L}$ was recorded at site 10 (well D0019), and an increase from 1,500 to 2,040 $\mu \mathrm{g} / \mathrm{L}$ at site 22 (well G0247).

The highest iron concentration $(4,550 \mathrm{ug} / \mathrm{L})$ measured was in the Pascagoula aquifer (site 54, well C0292). Water from the majority of samples from this aquifer had concentrations within the SDWR limit; with only four water samples exceeding the limit. Iron concentrations ranged from less than 2 to $4,550 \mu \mathrm{g} / \mathrm{L}$, with a median value of $10 \mu \mathrm{g} / \mathrm{L}$ (fig. 4). Since 1997, changes in iron concentration generally were small for most wells. The major exception to this is site 54 (well C0292), where iron concentrations increased from 600 to 4,550 $\mu \mathrm{g} / \mathrm{L}$ from 1999 to 2003.

Iron concentrations were low, ranging from 3 to $18 \mu \mathrm{g} / \mathrm{L}$, with a median value of $6.5 \mu \mathrm{g} / \mathrm{L}$, for wells screened in the Hattiesburg aquifer (fig. 4). For wells with multiple years of sampling, iron concentrations generally changed little.

In 2001, an iron concentration of $26 \mu \mathrm{g} / \mathrm{L}$ was measured in well J0211, which is screened in the Catahoula aquifer. 


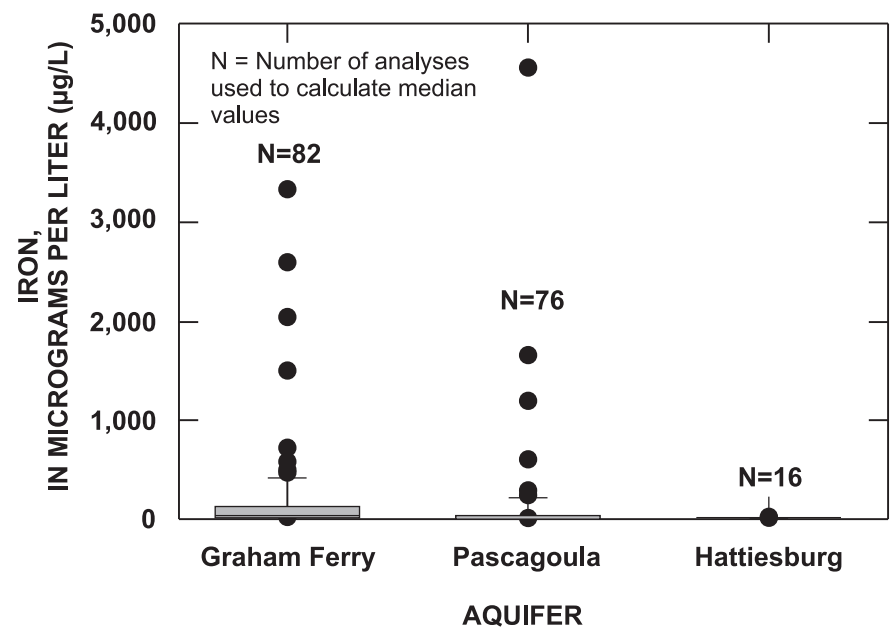

Figure 4. Distributions of iron concentrations in the Graham Ferry, Pascagoula, and Hattiesburg aquifers of Harrison County, Mississippi, 1997-2005.

\section{Manganese}

Manganese concentrations in the Citronelle aquifer were determined for two wells. Each well was sampled twice, and manganese concentrations ranged from 6 to $48 \mu \mathrm{g} / \mathrm{L}$, with a median value of $27 \mu \mathrm{g} / \mathrm{L}$. Differences in manganese concentrations during resampling were statistically insignificant.

Manganese concentrations ranged from less than 2 to $297 \mu \mathrm{g} / \mathrm{L}$, with a median value of $30 \mu \mathrm{g} / \mathrm{L}$, for the wells screened in the Graham Ferry aquifer (fig. 5). Concentrations exceeded the SDWR for manganese in 30 wells. For wells that were sampled multiple times since 1997, changes in manganese concentration were statistically insignificant.

The highest manganese concentrations were from wells screened in the Pascagoula aquifer (fig. 5). Manganese concentrations ranged from 0.8 to $382 \mu \mathrm{g} / \mathrm{L}$, with a median value of $8.25 \mu \mathrm{g} / \mathrm{L}$. Water in 12 wells exceeded the SDWR for manganese. For most of the wells sampled in multiple years,

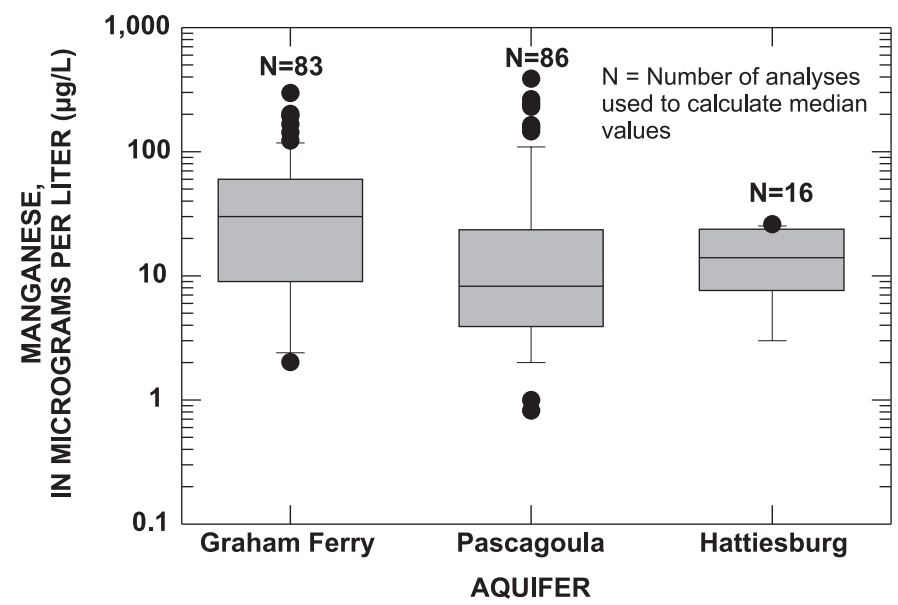

Figure 5. Distribution of manganese concentrations in the Graham Ferry, Pascagoula, and Hattiesburg aquifers of Harrison County, Mississippi, 1997-2005. the changes in manganese concentrations were statistically insignificant. However, a large increase in manganese concentration, from 20 to $382 \mu \mathrm{g} / \mathrm{L}$, occurred at site 54 (well C0292). Manganese concentrations ranged from 3 to $26 \mu \mathrm{g} / \mathrm{L}$, with a median value of $14 \mu \mathrm{g} / \mathrm{L}$, for wells screened in the Hattiesburg aquifer (fig. 5). The SDWR for manganese was not exceeded in water from any of the sampled wells. For wells with multiple years of samples, manganese concentrations changed little with time.

In 2001, a manganese concentration of $30 \mu \mathrm{g} / \mathrm{L}$ was measured at site 115 (well J0211), screened in the Catahoula aquifer.

\section{REFERENCES}

Strom, E.W. and Oakley, W.T., 1997, Water-quality data for selected wells in Harrison County, Mississippi, April 1997: U.S. Geological Survey Open-File Report 97-595, 21 p.

Strom, E.W. and Oakley, W.T., 1998, Water-quality data for selected wells in Harrison County, Mississippi, April 1998: U.S. Geological Survey Open-File Report 98-386, 7 p.

U.S. Environmental Protection Agency, 2004, 2004 Edition of the drinking water standards and health advisories: U.S. Environmental Protection Agency, EPA 822-R-04-005, accessed September 13, 2005 at URL http://www.epa.gov, waterscience/criteria/drinking/standards/dwstandards.pdd

U.S. Geological Survey, 1997, Water-level measurement using graduated steel tape: U.S. Geological Survey, Office of Ground Water Procedure document 1, 5 p.

Wilde, F.D., and Radtke, D.B., 1998, National field manual for the collection of water-quality data: U.S. Geological Survey Techniques of Water-Resources Investigations, book 9, variously paginated. 
Table 1. Selected site information for wells sampled in Harrison County, Mississippi, 1997-2005

[--, indicates no data available; -, indicates well flows without a pump; CTHL, Catahoula; CRNL, Citronelle; GRMF, Graham Ferry; HBRG, Hattiesburg; PCGL, Pascagoula; LSD, Land Surface Datum; GPM, gallons per minute; P, public supply; U, unused; T, Institution; I, irrigation; R, recreation; N, industrial; S, stock;

F, fire protection; Q, aquaculture]

\begin{tabular}{|c|c|c|c|c|c|c|c|c|}
\hline $\begin{array}{c}\text { Well } \\
\text { ref } \\
\text { number } \\
\text { (fig. 1) }\end{array}$ & $\begin{array}{l}\text { USGS } \\
\text { well } \\
\text { number }\end{array}$ & $\begin{array}{l}\text { Section, } \\
\text { township, } \\
\text { range }\end{array}$ & $\begin{array}{l}\text { Well } \\
\text { depth } \\
\text { (feet) }\end{array}$ & Aquifer & $\begin{array}{c}\text { Water } \\
\text { level } \\
\text { (feet } \\
\text { below LSD) }\end{array}$ & $\begin{array}{l}\text { Water- } \\
\text { level } \\
\text { date }\end{array}$ & $\begin{array}{c}\text { E-log } \\
\text { available }\end{array}$ & $\begin{array}{l}\text { Well } \\
\text { use }\end{array}$ \\
\hline 1 & E0109 & SWNWS32T06SR13W & 84 & CRNL & 7 & $2 / 1 / 1982$ & -- & $\mathrm{H}$ \\
\hline 2 & K0246 & SESES21T07SR12W & 70 & CRNL & 22.74 & $4 / 8 / 1998$ & -- & $\mathrm{H}$ \\
\hline 3 & A0033 & NWSWS35T05SR13W & 515 & GRMF & 97 & 4/1/1974 & -- & $\mathrm{H}$ \\
\hline 4 & A0047 & NESWS20T05SR13W & 410 & GRMF & 100 & $10 / 18 / 1991$ & -- & $\mathrm{H}$ \\
\hline 5 & A0049 & NESWS20T05NR13W & 220 & GRMF & 110 & $6 / 2 / 1988$ & -- & $\mathrm{H}$ \\
\hline 6 & A0056 & NESWS07T05SR13W & 184 & GRMF & 110 & $7 / 12 / 1980$ & -- & $\mathrm{H}$ \\
\hline 7 & В0012 & NESES35T04SR12W & 230 & GRMF & -- & -- & -- & $\mathrm{H}$ \\
\hline 8 & В0221 & SENWS20T05SR12W & 430 & GRMF & 106.95 & $4 / 6 / 1998$ & -- & $\mathrm{H}$ \\
\hline 9 & $\mathrm{C} 0019$ & SENES11T05SR11W & 230 & GRMF & 167.97 & $4 / 17 / 2000$ & -- & $\mathrm{H}$ \\
\hline 10 & D0019 & SWSWS21T05SR10W & 326 & GRMF & 86 & $12 / 1 / 1962$ & -- & $\mathrm{H}$ \\
\hline 11 & D0021 & SESES32T05SR09W & 252 & GRMF & 28 & $5 / 1 / 1971$ & -- & \\
\hline 12 & D0047 & SWNWS31T05S R09W & 300 & GRMF & 75.65 & $4 / 14 / 1999$ & -- & $\mathrm{H}$ \\
\hline 13 & D0049 & NWNES19T05SR10W & 92 & GRMF & 44 & $4 / 18 / 1977$ & -- & $\mathrm{H}$ \\
\hline 14 & D0052 & NESWS05T05SR09W & 277 & GRMF & 40 & $11 / 12 / 1975$ & -- & $\mathrm{H}$ \\
\hline 15 & E0099 & NENWS12T06SR13W & 420 & GRMF & 114.5 & 4/23/1997 & -- & $\mathrm{H}$ \\
\hline 16 & E0105 & NWNWS09T06SR13W & 245 & GRMF & 40 & $2 / 22 / 1979$ & -- & $\mathrm{H}$ \\
\hline 17 & E0119 & NENES35T06SR13W & 470 & GRMF & 60 & 8/10/1994 & -- & $\mathrm{H}$ \\
\hline 18 & E0120 & NENES11T06SR13W & 600 & GRMF & -- & -- & -- & $\mathrm{H}$ \\
\hline 19 & E0126 & SENES14T06SR13W & 550 & GRMF & -- & -- & -- & $\mathrm{H}$ \\
\hline 20 & F0375 & NENES28T06SR12W & 560 & GRMF & 80 & 7/11/1985 & -- & $\mathrm{H}$ \\
\hline 21 & F0406 & SWNWS19T06SR12W & 270 & GRMF & 60 & 4/1/1998 & -- & $\mathrm{H}$ \\
\hline 22 & G0247 & NWNWS01T06SR11W & 500 & GRMF & 158.77 & $4 / 9 / 1998$ & YES & $\mathrm{H}$ \\
\hline 23 & G0457 & NWSWSES14T06SR11W & 450 & GRMF & 24.74 & 4/13/1999 & YES & I \\
\hline 24 & H0279 & SWSES05T06SR10W & 490 & GRMF & 129.06 & $4 / 18 / 2000$ & YES & $\mathrm{R}$ \\
\hline 25 & H0321 & NWNES07T06S R09W & 330 & GRMF & 40 & $11 / 4 / 1980$ & -- & $\mathrm{H}$ \\
\hline 26 & H0403 & SWNWS09T06SR10W & 540 & GRMF & 131.56 & $4 / 10 / 1998$ & -- & $\mathrm{H}$ \\
\hline 27 & H0440 & SWNWS09T06SR10W & 63 & GRMF & -- & -- & -- & $\mathrm{H}$ \\
\hline 28 & J0132 & NESES15T07SR13W & 357 & GRMF & 94.76 & $4 / 7 / 1998$ & -- & $\mathrm{H}$ \\
\hline 29 & J0172 & NWNES09T07SR13W & 430 & GRMF & 97 & 9/1/1973 & -- & $\mathrm{H}$ \\
\hline 30 & J0210 & SWNES16T07SR13W & 435 & GRMF & 95 & $12 / 19 / 1986$ & -- & $\mathrm{H}$ \\
\hline 31 & K0258 & NENES09T07SR12W & 570 & GRMF & 72.73 & 4/8/1998 & -- & $\mathrm{H}$ \\
\hline 32 & K0329 & S21T07NR12W & 310 & GRMF & -- & -- & -- & I \\
\hline 33 & K0388 & NESWS21T07SR12W & 600 & GRMF & 60 & $6 / 13 / 2003$ & -- & S \\
\hline 34 & L0084 & NWNES26T07SR11W & 645 & GRMF & 57.18 & $12 / 30 / 1986$ & -- & $\mathrm{P}$ \\
\hline 35 & L0452 & NWSES23T07SR11W & 560 & GRMF & 68.55 & $4 / 18 / 2000$ & -- & I \\
\hline 36 & L0701 & SESWS16T07SR11W & 829 & GRMF & 52 & 5/4/1995 & YES & $\mathrm{P}$ \\
\hline
\end{tabular}


Table 1. Selected site information for wells sampled in Harrison County, Mississippi, 1997-2005--Continued

\begin{tabular}{|c|c|c|c|c|c|c|c|c|}
\hline $\begin{array}{c}\text { Well } \\
\text { ref } \\
\text { number } \\
\text { (fig. 1) }\end{array}$ & $\begin{array}{c}\text { USGS } \\
\text { well } \\
\text { number }\end{array}$ & $\begin{array}{l}\text { Section, } \\
\text { township, } \\
\text { range }\end{array}$ & $\begin{array}{l}\text { Well } \\
\text { depth } \\
\text { (feet) }\end{array}$ & Aquifer & $\begin{array}{c}\text { Water } \\
\text { level } \\
\text { (feet } \\
\text { below LSD) }\end{array}$ & $\begin{array}{l}\text { Water- } \\
\text { level } \\
\text { date }\end{array}$ & $\begin{array}{c}\text { E-log } \\
\text { available }\end{array}$ & $\begin{array}{l}\text { Well } \\
\text { use }\end{array}$ \\
\hline 37 & L0702 & SWSES10T07SR11W & 560 & GRMF & 50 & 9/14/1987 & -- & $\mathrm{H}$ \\
\hline 38 & L0725 & SENES06T07SR11W & 710 & GRMF & 87.65 & $4 / 18 / 2000$ & YES & $\mathrm{P}$ \\
\hline 39 & M0399 & NESWS05T07SR10W & 528 & GRMF & 30 & $3 / 1 / 1972$ & -- & $\mathrm{P}$ \\
\hline 40 & M0740 & SWNES34T07SR10W & 503 & GRMF & 50 & $12 / 17 / 1992$ & -- & $\mathrm{H}$ \\
\hline 41 & M0753 & S31T07SR09W & 680 & GRMF & 71.07 & $4 / 25 / 1997$ & YES & $\mathrm{P}$ \\
\hline 42 & M0800 & NESWS34T07SR10W & 680 & GRMF & -- & -- & -- & I \\
\hline 43 & N0333 & SENWS16T08SR13W & 700 & GRMF & 11.35 & 4/7/1998 & YES & $\mathrm{P}$ \\
\hline 44 & N0337 & SWNWS08T08SR13W & 357 & GRMF & 4 & 6/21/1977 & -- & $\mathrm{H}$ \\
\hline 45 & O0011 & SESES03T08SR12W & 590 & GRMF & 19.75 & $10 / 20 / 1982$ & -- & $\mathrm{P}$ \\
\hline 46 & O0308 & SESES02T08SR12W & 764 & GRMF & 29.63 & $4 / 22 / 1997$ & YES & $P$ \\
\hline 47 & O0319 & NWSWS08T08SR12W & 467 & GRMF & 18.91 & $4 / 7 / 1998$ & -- & $\mathrm{P}$ \\
\hline 48 & В0229 & NWSWS36T05SR12W & 600 & PCGL & 60 & $7 / 3 / 1985$ & -- & $\mathrm{H}$ \\
\hline 49 & В0245 & SENWS35T05SR12W & 706 & PCGL & 70.77 & $4 / 17 / 2000$ & -- & $\mathrm{P}$ \\
\hline 50 & $\mathrm{C} 0146$ & SWSES14T05SR11W & 476 & PCGL & 150 & $8 / 16 / 1976$ & -- & $\mathrm{H}$ \\
\hline 51 & $\mathrm{C} 0240$ & NESENES32T05SR $11 \mathrm{~W}$ & 635 & PCGL & 40 & $6 / 7 / 1975$ & -- & $\mathrm{H}$ \\
\hline 52 & $\mathrm{C} 0286$ & NWSWS35T04SR11W & 730 & PCGL & 120 & $8 / 28 / 1987$ & -- & $\mathrm{H}$ \\
\hline 53 & $\mathrm{C} 0288$ & SENES11T05SR11W & 645 & PCGL & 150 & $10 / 1 / 1988$ & YES & $\mathrm{H}$ \\
\hline 54 & $\mathrm{C} 0292$ & NWNES11T05SR11W & 515 & PCGL & -- & -- & -- & $\mathrm{H}$ \\
\hline 55 & $\mathrm{C} 0327$ & SWNES17T05SR11W & 810 & PCGL & 79 & 8/23/1997 & -- & $\mathrm{H}$ \\
\hline 56 & D0026 & NESES32T05SR10W & 705 & PCGL & 72 & $7 / 1 / 1973$ & -- & $\mathrm{H}$ \\
\hline 57 & D0045 & SES04T05SR09W & 620 & PCGL & 40 & $11 / 6 / 1980$ & -- & $\mathrm{H}$ \\
\hline 58 & E0125 & SESES12T06SR13W & 638 & PCGL & 119.38 & $4 / 16 / 1998$ & -- & $\mathrm{H}$ \\
\hline 59 & F0402 & NWNES23T06SR12W & 650 & PCGL & -- & -- & -- & $\mathrm{U}$ \\
\hline 60 & F0405 & SWNWS19T06SR12W & 950 & PCGL & 100 & $4 / 16 / 1998$ & YES & $\mathrm{T}$ \\
\hline 61 & G0005 & SWSWS16T06SR11W & 758 & PCGL & 47.63 & 4/9/1998 & -- & $\mathrm{P}$ \\
\hline 62 & H0048 & SESES20T06SR09W & 840 & PCGL & -5.54 & $4 / 24 / 1997$ & -- & $\mathrm{H}$ \\
\hline 63 & H0288 & SWNES17T06SR09W & 537 & PCGL & 30 & $4 / 7 / 1995$ & -- & $\mathrm{H}$ \\
\hline 64 & H0291 & SWSWS01T06SR10W & 640 & PCGL & 3 & 1/27/1975 & -- & $\mathrm{H}$ \\
\hline 65 & H0302 & NESWS29T06SR09W & 745 & PCGL & 35 & $11 / 21 / 1977$ & -- & $\mathrm{H}$ \\
\hline 66 & H0308 & NENES24T06SR10W & 882 & PCGL & 54.88 & $4 / 14 / 1999$ & -- & $\mathrm{H}$ \\
\hline 67 & J0219 & SWSES25T07SR13W & 940 & PCGL & 62.24 & $4 / 17 / 1998$ & -- & $\mathrm{H}$ \\
\hline 68 & J0290 & SESES26T07SR13W & 935 & PCGL & 55 & $6 / 1 / 1997$ & -- & $\mathrm{R}$ \\
\hline 69 & K0324 & SENWS04T07SR12W & 950 & PCGL & 50 & 9/20/1990 & -- & $\mathrm{H}$ \\
\hline 70 & K0327 & SENES24T07SR12W & 780 & PCGL & 44.43 & $4 / 9 / 1998$ & YES & $\mathrm{P}$ \\
\hline 71 & K0370 & NENES05T07SR12W & 680 & PCGL & -- & -- & -- & $\mathrm{H}$ \\
\hline 72 & K0382 & NWNWSWS10T07SR12W & 800 & PCGL & -- & -- & YES & $\mathrm{P}$ \\
\hline 73 & L0175 & S09T08SR11W & 1320 & PCGL & -9 & $9 / 1 / 1968$ & YES & $\mathrm{F}$ \\
\hline 74 & L0231 & SWNES04T07SR11W & 697 & PCGL & 62.23 & $10 / 22 / 1982$ & -- & $\mathrm{P}$ \\
\hline 75 & L0670 & SWSES09T07SR11W & 750 & PCGL & 56 & 7/9/1990 & -- & $\mathrm{P}$ \\
\hline
\end{tabular}


Table 1. Selected site information for wells sampled in Harrison County, Mississippi, 1997-2005--Continued

\begin{tabular}{|c|c|c|c|c|c|c|c|c|}
\hline $\begin{array}{c}\text { Well } \\
\text { ref } \\
\text { number } \\
\text { (fig. 1) }\end{array}$ & $\begin{array}{c}\text { USGS } \\
\text { well } \\
\text { number }\end{array}$ & $\begin{array}{l}\text { Section, } \\
\text { township, } \\
\text { range }\end{array}$ & $\begin{array}{l}\text { Well } \\
\text { depth } \\
\text { (feet) }\end{array}$ & Aquifer & $\begin{array}{c}\text { Water } \\
\text { level } \\
\text { (feet } \\
\text { below LSD) }\end{array}$ & $\begin{array}{l}\text { Water- } \\
\text { level } \\
\text { date }\end{array}$ & $\begin{array}{c}\text { E-log } \\
\text { available }\end{array}$ & $\begin{array}{l}\text { Well } \\
\text { use }\end{array}$ \\
\hline 76 & L0729 & SENES17T07SR11W & 805 & PCGL & 56.9 & $4 / 23 / 2000$ & -- & $\mathrm{H}$ \\
\hline 77 & M0266 & NWSES22T07SR09W & 1236 & PCGL & -17.56 & 4/24/1997 & -- & $\mathrm{H}$ \\
\hline 78 & M0643 & SESWS09T07SR09W & 840 & PCGL & 28.8 & $5 / 8 / 1985$ & YES & $\mathrm{P}$ \\
\hline 79 & M0657 & SWSWS31T07SR10W & 900 & PCGL & 50.75 & $12 / 30 / 1986$ & YES & $P$ \\
\hline 80 & M0734 & NES27T07SR09W & 1160 & PCGL & -- & -- & YES & $\mathrm{N}$ \\
\hline 81 & M0742 & SWSWS07T07SR09W & 900 & PCGL & 89.45 & $4 / 20 / 2000$ & -- & $\mathrm{H}$ \\
\hline 82 & M0749 & SWNES09T07SR09W & 860 & PCGL & 41 & $7 / 5 / 1996$ & -- & $\mathrm{P}$ \\
\hline 83 & M0751 & SWNES09T07SR10W & 805 & PCGL & 48.49 & $10 / 31 / 1996$ & YES & $\mathrm{P}$ \\
\hline 84 & M0752 & NENWS25T07SR09W & 965 & PCGL & 41 & 12/7/1995 & YES & I \\
\hline 85 & M0785 & S34T07SR09W & 740 & PCGL & 52.12 & $4 / 25 / 1997$ & YES & $\mathrm{P}$ \\
\hline 86 & M0790 & NWS33T07SR09W & 1300 & PCGL & -16 & $4 / 22 / 1997$ & -- & $\mathrm{N}$ \\
\hline 87 & M0791 & NWNWS03T07SR10W & 751 & PCGL & 55.24 & $4 / 9 / 1998$ & YES & $\mathrm{P}$ \\
\hline 88 & M0793 & SWSWS07T07SR09W & 1060 & PCGL & -- & -- & YES & $\mathrm{U}$ \\
\hline 89 & M0795 & SENES14T07SR09W & 900 & PCGL & 51.84 & $4 / 20 / 2000$ & YES & $\mathrm{P}$ \\
\hline 90 & M0812 & SES28T07SR10W & 950 & PCGL & 51 & $4 / 9 / 2003$ & -- & $\mathrm{P}$ \\
\hline 91 & N0287 & SWNWS33T08SR13W & 966 & PCGL & -15 & 9/2/1976 & YES & $\mathrm{P}$ \\
\hline 92 & N0313 & SWNWS34T08SR13W & 982 & PCGL & 9.16 & $4 / 19 / 2000$ & YES & $\mathrm{P}$ \\
\hline 93 & N0327 & NENWS25T08SR13W & 858 & PCGL & 6 & $4 / 23 / 1997$ & YES & $\mathrm{P}$ \\
\hline 94 & $\mathrm{O} 0123$ & NWNWS22T08SR12W & 873 & PCGL & 36 & $4 / 19 / 2000$ & YES & $\mathrm{P}$ \\
\hline 95 & O0296 & NESWS12T08SR12W & 862 & PCGL & 33 & $12--1986$ & YES & $\mathrm{P}$ \\
\hline 96 & $\mathrm{C} 0112$ & NWSWS20T05SR11W & 1140 & HBRG & 45 & $1 / 15 / 1975$ & YES & $\mathrm{H}$ \\
\hline 97 & $\mathrm{C} 0274$ & SWSWS06T05SR11W & 1412 & HBRG & 78 & $3 / 18 / 1985$ & YES & $\mathrm{P}$ \\
\hline 98 & $\mathrm{C} 0325$ & SENES33T05SR11W & 1414 & HBRG & 33.3 & 4/13/1999 & YES & $\mathrm{P}$ \\
\hline 99 & J0213 & S32T07SR13W & 1775 & HBRG & -13.86 & $4 / 23 / 1997$ & -- & $\mathrm{N}$ \\
\hline 100 & M0711 & NENES04T07SR10W & 1472 & HBRG & -- & -- & -- & $\mathrm{H}$ \\
\hline 101 & O0307 & NENES10T08SR12W & 1640 & HBRG & -37 & $4 / 22 / 1997$ & YES & $\mathrm{P}$ \\
\hline 102 & $\mathrm{O} 0320$ & NWSWS09T08SR12W & 1860 & HBRG & -37.4 & $4 / 7 / 1998$ & -- & Q \\
\hline 103 & $\mathrm{O} 0355$ & SESWS12T08SR12W & 1670 & HBRG & -27.5 & 4/7/1998 & YES & $\mathrm{P}$ \\
\hline 104 & J0211 & NENES27T07SR13W & 2413 & CTHL & -10.7 & $3 / 17 / 1988$ & YES & $\mathrm{H}$ \\
\hline
\end{tabular}


Appendix I. Water quality data for selected wells sampled from 1997 to 2005, Harrison County, Mississippi

[LSD, Land Surface Datum; Pt-Co, Platinum-Cobalt; uS/cm, Microsiemens per centimeter at 25 deg Celcius; mg/L, Milligrams per Liter; ug/L, Micrograms per Liter; <, less than; --, indicates no data available; E, estimated]

\begin{tabular}{|c|c|c|c|c|c|c|c|c|c|c|c|c|c|}
\hline $\begin{array}{c}\text { Well } \\
\text { refer- } \\
\text { ence } \\
\text { number } \\
\text { (fig. 1) }\end{array}$ & Station number & $\begin{array}{c}\text { Sample } \\
\text { date }\end{array}$ & $\begin{array}{c}\text { Well } \\
\text { depth } \\
\text { (feet } \\
\text { below } \\
\text { LSD) }\end{array}$ & $\begin{array}{c}\text { Color, } \\
\text { (Pt-Co } \\
\text { units) }\end{array}$ & $\begin{array}{l}\text { pH, } \\
\text { field }\end{array}$ & $\begin{array}{l}\text { pH, } \\
\text { lab }\end{array}$ & $\begin{array}{c}\text { Specific } \\
\text { conduc- } \\
\text { tance, } \\
\text { lab } \\
\text { (pS/cm) }\end{array}$ & $\begin{array}{c}\text { Specific } \\
\text { conduc- } \\
\text { tance, } \\
\text { field } \\
\text { (pS/cm) }\end{array}$ & $\begin{array}{c}\text { Temper- } \\
\text { ature, } \\
\text { water } \\
(\operatorname{deg} \mathrm{C})\end{array}$ & $\begin{array}{l}\text { Chlor- } \\
\text { ide, } \\
\text { (mg/L) }\end{array}$ & $\begin{array}{c}\text { Total } \\
\text { dis- } \\
\text { solved } \\
\text { solids } \\
\text { (mg/L) }\end{array}$ & $\begin{array}{l}\text { Iron } \\
(\mu \mathrm{g} / \mathrm{L})\end{array}$ & $\begin{array}{c}\text { Mangan- } \\
\text { ese } \\
(\mu \mathrm{g} / \mathrm{L})\end{array}$ \\
\hline \multicolumn{14}{|c|}{ Citronelle Aquifer } \\
\hline \multirow[t]{2}{*}{1} & 302844089192001 & 04/13/99 & 84 & 5 & 4.8 & 5.8 & 23 & 27 & -- & 3.3 & 24 & 66 & 6 \\
\hline & 302844089192001 & 04/07/03 & 84 & $<5$ & -- & 6.4 & 21 & 25 & -- & 3.4 & 20 & 70 & 7 \\
\hline \multirow[t]{2}{*}{2} & 302506089103501 & 04/08/98 & 70 & $<5$ & -- & 8.2 & 202 & 200 & -- & 3.3 & 162 & 6 & 47 \\
\hline & 302506089103501 & $04 / 16 / 02$ & 70 & $<5$ & -- & 7.9 & 199 & 204 & -- & 3.3 & 150 & 8 & 48 \\
\hline \multicolumn{14}{|c|}{ Graham Ferry Aquifer } \\
\hline 3 & 303355089160801 & $04 / 18 / 02$ & 515 & $<5$ & -- & 6.2 & 49 & 54 & 22 & 3.5 & 49 & 3 & 2 \\
\hline \multirow[t]{2}{*}{4} & 303541089185801 & $04 / 12 / 99$ & 410 & 5 & 7.9 & 8.5 & 239 & 226 & -- & 5.3 & 146 & 6 & 30 \\
\hline & 303541089185801 & 04/07/03 & 410 & $<5$ & 8 & 8.6 & 233 & 233 & -- & 5.4 & 134 & 7 & 31 \\
\hline \multirow[t]{2}{*}{5} & 303546089190901 & 04/12/99 & 220 & 5 & 6.5 & 6.9 & 93 & 102 & -- & 3.2 & 115 & 57 & 6 \\
\hline & 303546089190901 & 04/07/03 & 220 & $<5$ & 7.1 & 6.8 & 100 & 104 & -- & 3.6 & 109 & 580 & 53 \\
\hline \multirow[t]{2}{*}{6} & 303724089200001 & 04/12/99 & 184 & 5 & 6.2 & 6.8 & 101 & 98 & -- & 3.7 & 133 & 40 & 11 \\
\hline & 303724089200001 & 04/07/03 & 184 & $<5$ & 6.9 & 7 & 98 & 99 & -- & 4.2 & 130 & 38 & 9 \\
\hline \multirow[t]{3}{*}{7} & 303911089090801 & $04 / 23 / 97$ & 230 & 20 & 6.9 & 7.1 & 102 & 99 & -- & -- & -- & -- & -- \\
\hline & 303911089090801 & 04/09/01 & 230 & $<5$ & 5.5 & 6.9 & 105 & 101 & 20.5 & 2.8 & 120 & 519 & 50 \\
\hline & 303911089090801 & $04 / 11 / 05$ & 230 & 15 & 6.9 & 6.8 & 97 & 103 & -- & 2.6 & 103 & 472 & 47.8 \\
\hline \multirow[t]{2}{*}{8} & 303554089125401 & 04/06/98 & 430 & $<5$ & -- & 7 & 104 & 104 & -- & 3.2 & 110 & 260 & 18 \\
\hline & 303554089125401 & $04 / 15 / 02$ & 430 & $<5$ & 7 & 6.6 & 102 & 108 & 22 & 3.3 & 110 & 228 & 18 \\
\hline \multirow[t]{2}{*}{9} & 303732089032101 & $04 / 17 / 00$ & 230 & $<5$ & 8.8 & 8.4 & 274 & 281 & -- & 2.5 & 185 & 30 & 17 \\
\hline & 303732089032101 & $04 / 26 / 04$ & 230 & 10 & 8 & 8.4 & 280 & 291 & 23 & 2.2 & 190 & 55 & 16 \\
\hline \multirow[t]{3}{*}{10} & 303530088595201 & $04 / 23 / 97$ & 326 & 10 & 7.3 & 7.2 & 128 & 131 & -- & -- & -- & -- & -- \\
\hline & 303530088595201 & 04/09/01 & 326 & 40 & 6.3 & 7.1 & 129 & 154 & 23 & 3.0 & 123 & 2600 & 190 \\
\hline & 303530088595201 & $04 / 11 / 05$ & 326 & 50 & 6.8 & 7 & 120 & 138 & -- & 2.8 & 125 & 3330 & 194 \\
\hline \multirow[t]{2}{*}{11} & 303812089011001 & 04/17/00 & 252 & $<5$ & 8.7 & 8.4 & 282 & 288 & 23 & 3.3 & 166 & 10 & 5 \\
\hline & 303812089011001 & $04 / 26 / 04$ & 252 & 10 & 8.2 & 8.4 & 281 & 301 & 22.5 & 3.4 & 182 & 4 & 5 \\
\hline \multirow[t]{2}{*}{12} & 303402088555001 & 04/14/99 & 300 & 5 & 7.3 & 7.8 & 206 & 202 & -- & 2.8 & 162 & 125 & 53 \\
\hline & 303402088555001 & $04 / 09 / 03$ & 300 & $<5$ & 7.9 & 7.8 & 202 & 207 & -- & 3.0 & 166 & 151 & 54 \\
\hline \multirow[t]{2}{*}{13} & 303612089012901 & 04/13/99 & 92 & 5 & 5 & 5.3 & 20 & 28 & -- & 3.5 & 15 & 69 & 3 \\
\hline & 303612089012901 & 04/09/03 & 92 & $<5$ & 7.9 & 7.5 & 37720 & 57 & -- & 3.6 & 31 & 3 & 10 \\
\hline \multirow[t]{2}{*}{14} & 303820088543601 & 04/14/99 & 277 & 5 & 5.1 & 5.6 & 22 & 32 & -- & 2.8 & 25 & 5 & 3 \\
\hline & 303820088543601 & $04 / 09 / 03$ & 277 & $<5$ & -- & 6.7 & 21 & 27 & -- & 3.1 & 16 & 4 & 2 \\
\hline \multirow[t]{3}{*}{15} & 303354089161801 & $04 / 23 / 97$ & 420 & 10 & 7.4 & 7.9 & 198 & 151 & -- & -- & -- & -- & -- \\
\hline & 303354089161801 & 04/09/01 & 420 & $<5$ & 8.6 & 8.4 & 238 & 271 & 24 & 4.5 & 153 & 8 & 16 \\
\hline & 303354089161801 & $04 / 14 / 05$ & 420 & 2 & 8.4 & 8.6 & 223 & 240 & -- & 4.4 & 144 & 21 & 16.6 \\
\hline 16 & 303235089182501 & 04/13/99 & 245 & 5 & 6.9 & 7.7 & 169 & 165 & -- & 3.2 & 141 & 45 & 70 \\
\hline
\end{tabular}


Appendix I. Water quality data for selected wells sampled from 1997 to 2005, Harrison County, Mississippi--Continued

\begin{tabular}{|c|c|c|c|c|c|c|c|c|c|c|c|c|c|}
\hline $\begin{array}{l}\text { Well } \\
\text { refer- } \\
\text { ence } \\
\text { number } \\
\text { (fig. 1) }\end{array}$ & Station number & $\begin{array}{l}\text { Sample } \\
\text { date }\end{array}$ & $\begin{array}{l}\text { Well } \\
\text { depth } \\
\text { (feet } \\
\text { below } \\
\text { LSD) }\end{array}$ & $\begin{array}{l}\text { Color, } \\
\text { (Pt-Co } \\
\text { units) }\end{array}$ & $\begin{array}{l}\mathrm{pH}, \\
\text { field }\end{array}$ & $\begin{array}{l}\mathrm{pH}, \\
\text { lab }\end{array}$ & $\begin{array}{c}\text { Specific } \\
\text { conduc- } \\
\text { tance, } \\
\text { lab } \\
(\mu \mathrm{S} / \mathrm{cm})\end{array}$ & $\begin{array}{c}\text { Specific } \\
\text { conduc- } \\
\text { tance, } \\
\text { field } \\
(\mu \mathrm{S} / \mathrm{cm})\end{array}$ & $\begin{array}{c}\text { Temper- } \\
\text { ature, } \\
\text { water } \\
(\operatorname{deg} \mathrm{C})\end{array}$ & $\begin{array}{l}\text { Chlor- } \\
\text { ide, } \\
\text { (mg/L) }\end{array}$ & $\begin{array}{c}\text { Total } \\
\text { dis- } \\
\text { solved } \\
\text { solids } \\
\text { (mg/L) }\end{array}$ & $\begin{array}{c}\text { Iron } \\
\text { ( } \mu \mathrm{g} / \mathrm{L})\end{array}$ & $\begin{array}{c}\text { Mangan- } \\
\text { ese } \\
(\mu \mathrm{g} / \mathrm{L})\end{array}$ \\
\hline & 303235089182501 & $04 / 07 / 03$ & 245 & $<5$ & 7.6 & 7.9 & 168 & 172 & -- & 3.6 & 131 & 113 & 70 \\
\hline \multirow[t]{2}{*}{17} & 302910089153401 & 04/13/99 & 470 & 5 & -- & 7.2 & 170 & 168 & -- & 3.0 & 144 & 233 & 140 \\
\hline & 302910089153401 & $04 / 07 / 03$ & 470 & $<5$ & 6.8 & 7.5 & 171 & 176 & -- & 3.4 & 136 & 721 & 160 \\
\hline 18 & 303240089152801 & 04/08/98 & 600 & 20 & 7.3 & 7.4 & 158 & 156 & -- & 3.4 & 142 & 150 & 120 \\
\hline \multirow[t]{2}{*}{19} & 303132089153501 & $04 / 18 / 00$ & 550 & $<5$ & 7.1 & 7.3 & 173 & 171 & -- & 3.2 & 131 & 111 & 55 \\
\hline & 303132089153501 & $04 / 28 / 04$ & 550 & 5 & 7.3 & 7.6 & 172 & 180 & 22.5 & 3.4 & 148 & 61 & 59 \\
\hline \multirow[t]{2}{*}{20} & 302958089112401 & $04 / 08 / 98$ & 560 & 5 & 8.5 & 8.4 & 251 & 250 & -- & 4.0 & 174 & 7 & 2 \\
\hline & 302958089112401 & $04 / 15 / 02$ & 560 & $<5$ & 8.4 & 8.4 & 250 & 257 & 23.5 & 4.1 & 185 & 10 & 2 \\
\hline 21 & 303035089141001 & $04 / 16 / 98$ & 270 & $<5$ & 7.2 & 6.9 & 122 & 123 & -- & -- & 104 & 20 & 4 \\
\hline \multirow[t]{2}{*}{22} & 303329089025801 & $04 / 09 / 98$ & 500 & 20 & 7.5 & 7.3 & 123 & 131 & -- & 3.0 & 124 & 1500 & 59 \\
\hline & 303329089025801 & $04 / 18 / 02$ & 500 & 20 & 7.3 & 7 & 124 & 133 & -- & 3.0 & 130 & 2040 & 44 \\
\hline \multirow[t]{2}{*}{23} & 303104089034001 & $04 / 13 / 99$ & 450 & 10 & 8.2 & 8.6 & 330 & 318 & 23.5 & 2.7 & 219 & 44 & 18 \\
\hline & 303104089034001 & $04 / 26 / 04$ & 450 & 10 & 8.5 & 8.5 & 326 & 334 & 23.5 & 2.5 & 219 & 27 & 16 \\
\hline \multirow[t]{2}{*}{24} & 303244089003601 & $04 / 18 / 00$ & 490 & $<5$ & 8.1 & 8.3 & 322 & 328 & 23 & 2.2 & 211 & 43 & 35 \\
\hline & 303244089003601 & $04 / 26 / 04$ & 490 & 10 & 7.7 & 8.3 & 324 & 330 & 23.5 & 2.3 & 211 & 52 & 38 \\
\hline \multirow[t]{2}{*}{25} & 303237088552401 & $04 / 14 / 99$ & 330 & 5 & 8.2 & 8.5 & 319 & 307 & -- & 15 & 206 & 7 & 4 \\
\hline & 303237088552401 & $04 / 08 / 03$ & 330 & $<5$ & 8.4 & 8.5 & 303 & 308 & -- & 13 & 214 & 7 & 4 \\
\hline \multirow[t]{2}{*}{26} & 303216088595701 & $04 / 10 / 98$ & 540 & $<5$ & 7.7 & 7.9 & 268 & 272 & -- & 2.5 & 194 & 70 & 97 \\
\hline & 303216088595701 & $04 / 18 / 02$ & 540 & $<5$ & 7.7 & 7.7 & 265 & 269 & -- & 2.5 & 188 & 68 & 89 \\
\hline 27 & 303216088595602 & $04 / 18 / 02$ & 63 & $<5$ & 6.2 & 6.7 & 24 & 28 & -- & 4.3 & 18 & 18 & 9 \\
\hline \multirow[t]{2}{*}{28} & 302600089153301 & $04 / 07 / 98$ & 357 & 5 & 7.4 & 7.7 & 201 & 201 & -- & 3.3 & 166 & 210 & 70 \\
\hline & 302600089153301 & $04 / 16 / 02$ & 357 & $<5$ & 7.6 & 7.5 & 201 & 205 & -- & 3.3 & 156 & 57 & 51 \\
\hline \multirow[t]{2}{*}{29} & 302745089174501 & $04 / 07 / 98$ & 430 & 10 & 7.5 & 7.7 & 204 & 206 & -- & 3.5 & 164 & 260 & 100 \\
\hline & 302745089174501 & $04 / 16 / 02$ & 430 & $<5$ & 7.3 & 7.3 & 201 & 205 & -- & 3.5 & 167 & 319 & 100 \\
\hline \multirow[t]{2}{*}{30} & 302610089173401 & $04 / 19 / 00$ & 435 & $<5$ & 7.4 & 7.5 & 202 & 205 & 23 & 3.4 & 158 & 94 & 110 \\
\hline & 302610089173401 & $04 / 28 / 04$ & 435 & 5 & 7.5 & 7.8 & 170 & 178 & 20 & 5.0 & 150 & 61 & 114 \\
\hline \multirow[t]{2}{*}{31} & 302651089113801 & $04 / 08 / 98$ & 570 & $<5$ & 7.4 & 7.6 & 192 & 191 & -- & 3.4 & 160 & 10 & 63 \\
\hline & 302651089113801 & $04 / 16 / 02$ & 570 & $<5$ & 7.5 & 7.4 & 191 & 192 & 24 & 3.3 & 154 & 32 & 69 \\
\hline 32 & 302506089113601 & $04 / 19 / 00$ & 310 & $<5$ & 7.9 & 7.8 & 212 & 216 & 23.5 & 3.1 & 155 & 34 & 37 \\
\hline 33 & 302503089115301 & 05/03/04 & 600 & 8 & 7.5 & 7.9 & 221 & 229 & 23 & 3.0 & 168 & 115 & 297 \\
\hline \multirow[t]{3}{*}{34} & 302440089033001 & $04 / 24 / 97$ & 645 & 10 & 8.1 & 8.2 & 246 & 245 & 24 & 3.5 & 180 & -- & -- \\
\hline & 302440089033001 & $04 / 11 / 01$ & 645 & $<5$ & 8.1 & 8.2 & 246 & 239 & 25 & 3.2 & 173 & 31 & 16 \\
\hline & 302440089033001 & $04 / 12 / 05$ & 645 & 2 & 7.8 & 8.2 & 227 & 249 & 24.5 & 3.1 & 174 & 60 & 17.7 \\
\hline
\end{tabular}


Appendix I. Water quality data for selected wells sampled from 1997 to 2005, Harrison County, Mississippi--Continued

\begin{tabular}{|c|c|c|c|c|c|c|c|c|c|c|c|c|c|}
\hline $\begin{array}{l}\text { Well } \\
\text { refer- } \\
\text { ence } \\
\text { number } \\
\text { (fig. 1) }\end{array}$ & Station number & $\begin{array}{c}\text { Sample } \\
\text { date }\end{array}$ & $\begin{array}{l}\text { Well } \\
\text { depth } \\
\text { (feet } \\
\text { below } \\
\text { LSD) }\end{array}$ & $\begin{array}{l}\text { Color, } \\
\text { (Pt-Co } \\
\text { units) }\end{array}$ & $\begin{array}{l}\mathrm{pH}, \\
\text { field }\end{array}$ & $\begin{array}{l}\mathrm{pH}, \\
\text { lab }\end{array}$ & $\begin{array}{c}\text { Specific } \\
\text { conduc- } \\
\text { tance, } \\
\text { lab } \\
(\mu \mathrm{S} / \mathrm{cm})\end{array}$ & $\begin{array}{c}\text { Specific } \\
\text { conduc- } \\
\text { tance, } \\
\text { field } \\
(\mu \mathrm{S} / \mathrm{cm})\end{array}$ & $\begin{array}{c}\text { Temper- } \\
\text { ature, } \\
\text { water } \\
\text { (deg C) }\end{array}$ & $\begin{array}{l}\text { Chlor- } \\
\text { ide, } \\
\text { (mg/L) }\end{array}$ & $\begin{array}{l}\text { Total } \\
\text { dis- } \\
\text { solved } \\
\text { solids } \\
\text { (mg/L) }\end{array}$ & $\begin{array}{c}\text { Iron } \\
(\mu \mathrm{g} / \mathrm{L})\end{array}$ & $\begin{array}{c}\text { Mangan- } \\
\text { ese } \\
(\mu \mathrm{g} / \mathrm{L})\end{array}$ \\
\hline 35 & 302500089032601 & $04 / 18 / 00$ & 560 & $<5$ & 6.6 & 7.9 & 216 & 212 & 24.5 & 3.4 & 157 & 146 & 25 \\
\hline \multirow[t]{2}{*}{36} & 302545089054501 & 04/18/00 & 829 & $<5$ & 8.6 & 8.8 & 246 & 255 & 25 & 2.8 & 171 & 7 & 12 \\
\hline & 302545089054501 & $04 / 27 / 04$ & 829 & 5 & 8.1 & 8.5 & 250 & 260 & 26 & 2.9 & 176 & 4 & 13 \\
\hline \multirow[t]{2}{*}{37} & 302642089043701 & 04/18/00 & 560 & $<5$ & 8.1 & 7.9 & 172 & 173 & 21.5 & 2.9 & 145 & 132 & 60 \\
\hline & 302642089043701 & $04 / 29 / 04$ & 560 & 5 & 7.3 & 8.1 & 171 & 176 & 23 & 3.1 & 148 & 142 & 64 \\
\hline \multirow[t]{2}{*}{38} & 302800089072001 & 04/18/00 & 710 & $<5$ & 8.1 & 8.2 & 229 & 233 & 25 & 2.7 & 169 & 45 & 34 \\
\hline & 302800089072001 & $04 / 27 / 04$ & 710 & 5 & 7.6 & 8.2 & 230 & 238 & 25 & 2.8 & 176 & 50 & 34 \\
\hline \multirow[t]{2}{*}{39} & 302745089004401 & $04 / 20 / 00$ & 528 & 5 & 6.8 & 7.7 & 173 & 177 & 23.5 & 2.9 & 140 & 275 & 12 \\
\hline & 302745089004401 & 05/03/04 & 528 & 7 & 7.6 & 7.8 & 173 & 176 & 24 & 3.0 & 147 & 299 & 14 \\
\hline \multirow[t]{2}{*}{40} & 302336088583201 & 04/16/99 & 503 & 10 & 9.2 & 8.9 & 330 & -- & -- & 2.8 & 215 & 25 & 2 \\
\hline & 302336088583201 & $04 / 10 / 03$ & 503 & $<5$ & 8.5 & 9 & 325 & 337 & -- & 3.0 & 212 & 27 & 2 \\
\hline \multirow[t]{3}{*}{41} & 302348088550301 & $04 / 25 / 97$ & 680 & 20 & 8.5 & 8.5 & 303 & 297 & -- & 5.3 & 194 & -- & -- \\
\hline & 302348088550301 & 04/10/01 & 680 & $<5$ & 8.3 & 8.3 & 322 & 309 & 26.5 & 6.1 & 207 & 13 & 30 \\
\hline & 302348088550301 & $04 / 13 / 05$ & 680 & 8 & 8.1 & 8.4 & 298 & 322 & 26.9 & 6.3 & 201 & 24 & 34.1 \\
\hline \multirow[t]{2}{*}{42} & 302334088583501 & 04/20/00 & 680 & $<5$ & 7.6 & 9.1 & 324 & 329 & 25 & 4.6 & 202 & 2 & 8 \\
\hline & 302334088583501 & $04 / 29 / 04$ & 680 & 10 & 9 & 9 & 323 & 334 & 25 & 4.6 & 203 & $<2$ & 8 \\
\hline \multirow[t]{2}{*}{43} & 302239089161001 & 04/07/98 & 700 & 20 & 8.9 & 9 & 530 & 529 & 25 & 16 & 326 & 10 & 12 \\
\hline & 302239089161001 & 04/19/02 & 700 & 10 & 8.6 & 9 & 540 & 554 & -- & 18 & 334 & 10 & 12 \\
\hline \multirow[t]{2}{*}{44} & 302246089160201 & $04 / 19 / 00$ & 357 & $<5$ & 7.5 & 7.6 & 202 & 209 & 22.5 & 3.1 & 160 & 83 & 120 \\
\hline & 302246089160201 & $04 / 28 / 04$ & 357 & $<5$ & 7.8 & 8 & 203 & 210 & 21 & 3.4 & 162 & 87 & 120 \\
\hline \multirow[t]{3}{*}{45} & 302215089102001 & $04 / 22 / 97$ & 590 & 20 & 8 & 8 & 190 & 185 & -- & 3.1 & 152 & -- & -- \\
\hline & 302215089102001 & 04/10/01 & 590 & $<5$ & 8.1 & 8 & 193 & 185 & 24.5 & 2.9 & 154 & 67 & 5 \\
\hline & 302215089102001 & $04 / 12 / 05$ & 590 & $<1$ & 7.8 & 7.8 & 176 & 184 & 24.6 & 2.8 & 158 & 93 & 4.4 \\
\hline \multirow[t]{3}{*}{46} & 302217089091301 & $04 / 22 / 97$ & 764 & 10 & 8.4 & 8.3 & 247 & 240 & 26 & 4.1 & 176 & -- & -- \\
\hline & 302217089091301 & 04/10/01 & 764 & $<5$ & 8.1 & 8.3 & 246 & 240 & 26 & 3.2 & 176 & 14 & 57 \\
\hline & 302217089091301 & $04 / 12 / 05$ & 764 & 2 & 8.3 & 8.3 & 228 & 206 & -- & 3.0 & 182 & 14 & 59.9 \\
\hline \multirow[t]{2}{*}{47} & 302134089130201 & 04/07/98 & 467 & $<5$ & -- & 8 & 226 & 224 & -- & 3.5 & 198 & 8 & 2 \\
\hline & 302134089130201 & 04/19/02 & 467 & $<5$ & 7 & 8 & 227 & 223 & -- & 3.5 & 190 & 7 & 2 \\
\hline \multicolumn{14}{|c|}{ Pascagoula Aquifer } \\
\hline \multirow[t]{2}{*}{48} & 303351089090901 & 04/06/98 & 600 & $<5$ & -- & 8.6 & 183 & -- & -- & 2.6 & 152 & 10 & 5 \\
\hline & 303351089090901 & $04 / 15 / 02$ & 600 & $<5$ & 8.6 & 8.5 & 183 & 188 & 22.5 & 2.5 & 144 & 2 & 5 \\
\hline \multirow[t]{2}{*}{49} & 303405089100001 & 04/17/00 & 706 & $<5$ & 8.8 & 8.7 & 213 & 218 & 25.5 & 2.3 & 145 & 13 & 15 \\
\hline & 303405089100001 & $04 / 26 / 04$ & 706 & 5 & 8.3 & 8.4 & 213 & 220 & 25 & 2.4 & 149 & 9 & 12 \\
\hline \multirow[t]{3}{*}{50} & 303540089024801 & 04/23/97 & 476 & 20 & 5.6 & 5.3 & 29 & 29 & -- & -- & -- & -- & -- \\
\hline & 303540089024801 & 04/09/01 & 476 & $<5$ & 5.2 & 5.2 & 30 & 24 & 21 & 6.5 & 21 & 6 & 3 \\
\hline & 303540089024801 & $04 / 11 / 05$ & 476 & 2 & 5 & 4.7 & 28 & 26 & -- & 5.2 & 16 & 26 & 2.5 \\
\hline
\end{tabular}


Appendix I. Water quality data for selected wells sampled from 1997 to 2005, Harrison County, Mississippi--Continued

\begin{tabular}{|c|c|c|c|c|c|c|c|c|c|c|c|c|c|}
\hline $\begin{array}{l}\text { Well } \\
\text { refer- } \\
\text { ence } \\
\text { number } \\
\text { (fig. 1) }\end{array}$ & Station number & $\begin{array}{c}\text { Sample } \\
\text { date }\end{array}$ & $\begin{array}{c}\text { Well } \\
\text { depth } \\
\text { (feet } \\
\text { below } \\
\text { LSD) }\end{array}$ & $\begin{array}{l}\text { Color, } \\
\text { (Pt-Co } \\
\text { units) }\end{array}$ & $\begin{array}{l}\mathrm{pH}, \\
\text { field }\end{array}$ & $\begin{array}{l}\mathrm{pH}, \\
\text { lab }\end{array}$ & $\begin{array}{c}\text { Specific } \\
\text { conduc- } \\
\text { tance, } \\
\text { lab } \\
(\mu S / \mathrm{cm})\end{array}$ & $\begin{array}{c}\text { Specific } \\
\text { conduc- } \\
\text { tance, } \\
\text { field } \\
(\mu \mathrm{S} / \mathrm{cm})\end{array}$ & $\begin{array}{l}\text { Temper- } \\
\text { ature, } \\
\text { water } \\
(\operatorname{deg} C)\end{array}$ & $\begin{array}{l}\text { Chlor- } \\
\text { ide, } \\
\text { (mg/L) }\end{array}$ & $\begin{array}{l}\text { Total } \\
\text { dis- } \\
\text { solved } \\
\text { solids } \\
\text { (mg/L) }\end{array}$ & $\begin{array}{l}\text { Iron } \\
\text { ( } \mu \mathrm{g} / \mathrm{L})\end{array}$ & $\begin{array}{c}\text { Mangan- } \\
\text { ese } \\
(\mu \mathrm{g} / \mathrm{L})\end{array}$ \\
\hline 51 & 303400089073301 & $04 / 06 / 98$ & 635 & 20 & -- & 7 & 105 & 109 & -- & 3.0 & 124 & 1200 & 42 \\
\hline 52 & 303851089043301 & $04 / 21 / 99$ & 730 & 5 & 8.5 & 8.9 & 308 & 295 & 22.5 & 2.5 & 191 & 3 & 9 \\
\hline 53 & 303736089032701 & $04 / 21 / 99$ & 645 & 5 & 8.3 & 8.6 & 301 & 292 & 23.5 & 2.5 & 200 & 11 & 8 \\
\hline \multirow[t]{2}{*}{54} & 303746089033501 & $04 / 21 / 99$ & 515 & 10 & 6.4 & 6.9 & 98 & 97 & 24.5 & 3.2 & 113 & 601 & 20 \\
\hline & 303746089033501 & $04 / 09 / 03$ & 515 & $<5$ & -- & 6.8 & 104 & -- & -- & 3.2 & 92 & 4550 & 382 \\
\hline \multirow[t]{2}{*}{55} & 303651089063401 & $04 / 21 / 99$ & 810 & 10 & 8.9 & 9.1 & 433 & 425 & 24.5 & 2.4 & 265 & 5 & 5 \\
\hline & 303651089063401 & $04 / 09 / 03$ & 810 & 40 & 9.2 & 9.2 & 413 & 422 & -- & 2.4 & 252 & 4 & 4 \\
\hline \multirow[t]{2}{*}{56} & 303335088593001 & $04 / 09 / 98$ & 705 & 40 & 8.9 & 8.9 & 594 & 592 & -- & 3.1 & 372 & 20 & 16 \\
\hline & 303335088593001 & $04 / 18 / 02$ & 705 & 20 & 8.6 & 8.9 & 601 & 611 & 24 & 3.2 & 370 & 5 & 8 \\
\hline \multirow[t]{2}{*}{57} & 303813088530801 & 04/14/99 & 620 & 5 & 8.4 & 9 & 255 & 251 & -- & 2.1 & 169 & 5 & 3 \\
\hline & 303813088530801 & $04 / 09 / 03$ & 620 & $<5$ & 8.6 & 9.1 & 254 & 262 & -- & 2.3 & 169 & 2 & 4 \\
\hline \multirow[t]{2}{*}{58} & 303155089143901 & $04 / 16 / 98$ & 638 & $<5$ & 7.4 & 7.3 & 175 & 177 & -- & 3.4 & 152 & 190 & 140 \\
\hline & 303155089143901 & $04 / 16 / 02$ & 638 & $<5$ & 7.2 & 7 & 174 & 168 & 23.5 & 3.3 & 140 & 276 & 96 \\
\hline 59 & 303037089093101 & $04 / 24 / 97$ & 650 & 20 & 7.6 & 7.6 & 172 & 175 & 24 & 3.7 & 142 & 120 & 63 \\
\hline \multirow[t]{2}{*}{60} & 303035089141101 & $04 / 16 / 98$ & 950 & 10 & 9.1 & 9.1 & 303 & 301 & 26 & 2.3 & 194 & 20 & 2 \\
\hline & 303035089141101 & $04 / 19 / 02$ & 950 & $<5$ & 8.7 & 9 & 300 & 308 & 25.5 & 2.3 & 187 & 11 & 1 \\
\hline \multirow[t]{2}{*}{61} & 303114089055601 & 04/09/98 & 758 & 5 & 9.2 & 9 & 282 & 283 & 26 & 2.4 & 176 & 20 & 2 \\
\hline & 303114089055601 & $04 / 15 / 02$ & 758 & $<5$ & 8.9 & 9 & 274 & 283 & 26 & 2.4 & 181 & 5 & 2 \\
\hline \multirow[t]{4}{*}{62} & 303009088540601 & $04 / 24 / 97$ & 840 & 20 & 8.6 & 8.8 & 946 & 911 & -- & 160 & 514 & -- & -- \\
\hline & 303009088540601 & $04 / 22 / 99$ & 840 & -- & 8.4 & 8.7 & 947 & 925 & 25 & 160 & -- & -- & -- \\
\hline & 303009088540601 & $04 / 12 / 01$ & 840 & 10 & 8.9 & 8.7 & 951 & 918 & 23.5 & 160 & 538 & 4 & 29 \\
\hline & 303009088540601 & $04 / 11 / 05$ & 840 & -- & 8.4 & 8.5 & 924 & 938 & -- & 157 & 531 & -- & -- \\
\hline \multirow[t]{2}{*}{63} & 303124089542501 & $04 / 22 / 99$ & 537 & 10 & 9.1 & 9.1 & 310 & 292 & 24.5 & -- & -- & 4 & 2 \\
\hline & 303124089542501 & $04 / 08 / 03$ & 537 & $<5$ & 9.1 & 9.1 & 299 & 302 & -- & 8.2 & 191 & 2 & 2 \\
\hline \multirow[t]{2}{*}{64} & 303217088570401 & $04 / 22 / 99$ & 640 & 10 & 9 & 9 & 279 & 272 & 25 & 6.1 & 180 & 3 & 3 \\
\hline & 303217088570401 & $04 / 08 / 03$ & 640 & $<5$ & 9.2 & 9.1 & 275 & 283 & -- & 6.6 & 181 & 2 & 3 \\
\hline \multirow[t]{2}{*}{65} & 302930088543401 & $04 / 22 / 99$ & 745 & 10 & 8.7 & 8.8 & 508 & 492 & 26 & 46 & 299 & 4 & 13 \\
\hline & 302930088543401 & $04 / 08 / 03$ & 745 & 10 & 8.8 & 9 & 487 & -- & -- & 43 & 291 & $<2$ & 12 \\
\hline \multirow[t]{2}{*}{66} & 303052088561001 & $04 / 14 / 99$ & 882 & 10 & 9.2 & 9 & 320 & 313 & 25 & 6.9 & 206 & 3 & 7 \\
\hline & 303052088561001 & $04 / 08 / 03$ & 882 & 30 & 8.5 & 9.1 & 307 & 309 & -- & 7.1 & 183 & 54 & 33 \\
\hline 67 & 303404089145001 & 04/07/98 & 940 & 10 & 9.1 & 9.1 & 405 & 398 & -- & 2.7 & 256 & 3 & 6 \\
\hline \multirow[t]{2}{*}{68} & 302403089153301 & $04 / 19 / 00$ & 935 & 5 & 8.1 & 9.1 & 417 & 428 & 26 & 2.6 & 261 & 4 & 10 \\
\hline & 302403089153301 & $04 / 27 / 04$ & 935 & 10 & 9 & 8.9 & 410 & 430 & 26.5 & 2.7 & 258 & 5 & 10 \\
\hline \multirow[t]{2}{*}{69} & 302741089124001 & $04 / 15 / 99$ & 950 & 10 & 9.4 & 9.1 & 363 & 357 & -- & 2.0 & 229 & 5 & 1 \\
\hline & 302741089124001 & 04/10/03 & 950 & $<5$ & 8.6 & 9.2 & 362 & 372 & -- & 2.2 & 218 & $<2$ & $<1$ \\
\hline
\end{tabular}


Appendix I. Water quality data for selected wells sampled from 1997 to 2005, Harrison County, Mississippi--Continued

\begin{tabular}{|c|c|c|c|c|c|c|c|c|c|c|c|c|c|}
\hline $\begin{array}{l}\text { Well } \\
\text { refer- } \\
\text { ence } \\
\text { number } \\
\text { (fig. 1) }\end{array}$ & Station number & $\begin{array}{c}\text { Sample } \\
\text { date }\end{array}$ & $\begin{array}{c}\text { Well } \\
\text { depth } \\
\text { (feet } \\
\text { below } \\
\text { LSD) }\end{array}$ & $\begin{array}{l}\text { Color, } \\
\text { (Pt-Co } \\
\text { units) }\end{array}$ & $\begin{array}{l}\mathrm{pH}, \\
\text { field }\end{array}$ & $\begin{array}{l}\text { pH, } \\
\text { lab }\end{array}$ & $\begin{array}{c}\text { Specific } \\
\text { conduc- } \\
\text { tance, } \\
\text { lab } \\
(\mu \mathrm{S} / \mathrm{cm})\end{array}$ & $\begin{array}{c}\text { Specific } \\
\text { conduc- } \\
\text { tance, } \\
\text { field } \\
(\mu \mathrm{S} / \mathrm{cm})\end{array}$ & $\begin{array}{c}\text { Temper- } \\
\text { ature, } \\
\text { water } \\
(\operatorname{deg} \mathrm{C})\end{array}$ & $\begin{array}{l}\text { Chlor- } \\
\text { ide, } \\
\text { (mg/L) }\end{array}$ & $\begin{array}{l}\text { Total } \\
\text { dis- } \\
\text { solved } \\
\text { solids } \\
\text { (mg/L) }\end{array}$ & $\begin{array}{c}\text { Iron } \\
\text { ( } \mu \mathrm{g} / \mathrm{L})\end{array}$ & $\begin{array}{c}\text { Mangan- } \\
\text { ese } \\
(\mu \mathrm{g} / \mathrm{L})\end{array}$ \\
\hline \multirow[t]{2}{*}{70} & 302518089082301 & 04/09/98 & 780 & 5 & 8.8 & 8.6 & 276 & 276 & 25 & 3.1 & 196 & 20 & 3 \\
\hline & 302518089082301 & $04 / 17 / 02$ & 780 & $<5$ & 8.5 & 8.7 & 274 & 278 & 25.5 & 3.2 & 188 & 24 & 4 \\
\hline \multirow[t]{2}{*}{71} & 302809089123401 & $04 / 15 / 99$ & 680 & 5 & 7.9 & 7.9 & 205 & 202 & -- & 3.4 & 163 & 52 & 240 \\
\hline & 302809089123401 & 04/10/03 & 680 & $<5$ & 8.1 & 8.1 & 201 & 206 & -- & 3.6 & 153 & 50 & 228 \\
\hline \multirow[t]{2}{*}{72} & 302656089111201 & $04 / 21 / 00$ & 800 & $<5$ & 7.9 & 8.5 & 273 & 275 & 24 & 2.7 & 186 & 18 & 19 \\
\hline & 302656089111201 & $04 / 28 / 04$ & 800 & 5 & 8.4 & 8.5 & 274 & 286 & 25.5 & 2.9 & 190 & 10 & 23 \\
\hline 73 & 302110089053301 & $04 / 15 / 99$ & 1320 & 40 & 9.1 & 8.9 & 781 & 779 & 28.5 & 8.3 & 494 & 13 & 14 \\
\hline \multirow[t]{3}{*}{74} & 302810089060002 & $04 / 24 / 97$ & 697 & 20 & 8.4 & 8.5 & 229 & 224 & 25 & 3.7 & 156 & -- & -- \\
\hline & 302810089060002 & $04 / 11 / 01$ & 697 & $<5$ & 8.4 & 8.5 & 227 & 220 & 25 & 3.4 & 169 & 6 & 20 \\
\hline & 302810089060002 & $04 / 12 / 05$ & 697 & $<1$ & 8.4 & 8.4 & 210 & 218 & 26.6 & 3.3 & 162 & 11 & 21.6 \\
\hline \multirow[t]{3}{*}{75} & 302646089053001 & $04 / 24 / 97$ & 750 & 10 & 8.4 & 8.5 & 240 & 237 & 25.5 & 3.5 & 170 & -- & -- \\
\hline & 302646089053001 & $04 / 11 / 01$ & 750 & $<5$ & 8.3 & 8.5 & 238 & 231 & 25.5 & 3.3 & 174 & 4 & 25 \\
\hline & 302646089053001 & $04 / 12 / 05$ & 750 & 2 & 7.8 & 8.4 & 223 & 249 & -- & 3.2 & 166 & 8 & 25.4 \\
\hline \multirow[t]{2}{*}{76} & 302605089062101 & $04 / 23 / 99$ & 805 & 5 & 8 & 8.3 & 260 & 258 & 26 & 2.8 & 187 & 63 & 69 \\
\hline & 302605089062101 & $04 / 10 / 03$ & 805 & $<5$ & 8.1 & 8.5 & 260 & 270 & -- & 2.8 & 185 & 10 & 54 \\
\hline \multirow[t]{5}{*}{77} & 302557088530301 & $04 / 24 / 97$ & 1236 & 30 & 8.6 & 8.7 & 1870 & 1800 & -- & 430 & 982 & -- & -- \\
\hline & 302557088530301 & $04 / 23 / 99$ & 1236 & -- & 9.9 & 9.5 & 1850 & 1790 & 23 & 430 & -- & -- & -- \\
\hline & 302557088530301 & $04 / 12 / 01$ & 1236 & 20 & 9.5 & 9.6 & 1840 & 1770 & 23 & 410 & 990 & $<2$ & 3 \\
\hline & 302557088530301 & $04 / 10 / 03$ & 1236 & 50 & 9.7 & 10 & 1840 & 1890 & -- & 420 & 952 & $<2$ & 2 \\
\hline & 302557088530301 & $04 / 13 / 05$ & 1236 & -- & 10.2 & 10.1 & 1800 & 1840 & -- & 419 & 970 & E3 & 0.8 \\
\hline \multirow[t]{3}{*}{78} & 302710088531501 & $04 / 24 / 97$ & 840 & 10 & 8.8 & 9 & 556 & 545 & 27 & 38 & 324 & -- & -- \\
\hline & 302710088531501 & 04/10/01 & 840 & 10 & 9 & 8.9 & 566 & 547 & 27.5 & 39 & 335 & $<2$ & -- \\
\hline & 302710088531501 & $04 / 13 / 05$ & 840 & 8 & 8.1 & 8.5 & 372 & 404 & -- & 25 & 245 & 21 & 4.5 \\
\hline \multirow[t]{3}{*}{79} & 302304089020301 & $04 / 24 / 97$ & 900 & 10 & 8.7 & 8.8 & 275 & 271 & -- & 4.1 & 180 & -- & -- \\
\hline & 302304089020301 & 04/11/01 & 900 & $<5$ & 8.9 & 8.8 & 273 & 268 & 26 & 3.8 & 184 & 4 & 8 \\
\hline & 302304089020301 & $04 / 12 / 05$ & 900 & 8 & 8.8 & 8.7 & 256 & 275 & -- & 3.5 & 173 & E5 & 8.5 \\
\hline 80 & 302438088531001 & $04 / 17 / 02$ & 1160 & 20 & 8.6 & 8.3 & 1810 & 1810 & -- & 410 & 988 & 63 & 33 \\
\hline \multirow[t]{2}{*}{81} & 302640088555301 & 04/20/00 & 900 & $<5$ & 7.3 & 7.6 & 195 & 204 & 24.5 & 3.3 & 158 & 228 & 150 \\
\hline & 302640088555301 & $04 / 28 / 04$ & 900 & 10 & 7.4 & 8.2 & 194 & 204 & 23 & 3.4 & 164 & 290 & 156 \\
\hline \multirow[t]{2}{*}{82} & 302708088532601 & $04 / 20 / 00$ & 860 & 5 & 8.5 & 9.1 & 379 & 386 & 27.5 & 14 & 232 & 4 & 14 \\
\hline & 302708088532601 & $04 / 29 / 04$ & 860 & 10 & 8.9 & 8.9 & 379 & 395 & 27 & 16 & 235 & $<2$ & 13 \\
\hline \multirow[t]{3}{*}{83} & 302701088593301 & $10 / 31 / 96$ & 805 & $<5$ & 9.3 & 9.2 & 325 & 316 & 27 & 5.0 & 210 & 4 & 2 \\
\hline & 302701088593301 & $04 / 12 / 01$ & 805 & 5 & 9.4 & 9.2 & 322 & 314 & 26.5 & 6.4 & 206 & 3 & 5 \\
\hline & 302701088593301 & $04 / 13 / 05$ & 805 & 8 & 8.6 & 8.8 & 304 & 330 & -- & 11 & 203 & 56 & 4.3 \\
\hline \multirow[t]{2}{*}{84} & 302445088563501 & $04 / 22 / 97$ & 965 & 20 & 9.2 & 9.2 & 346 & 342 & 28 & 5.0 & 208 & 8 & 3 \\
\hline & 302445088563501 & 04/10/01 & 965 & 10 & 9.1 & 9.2 & 348 & 342 & 27 & 6.6 & 219 & 7 & 5 \\
\hline
\end{tabular}


Appendix I. Water quality data for selected wells sampled from 1997 to 2005, Harrison County, Mississippi--Continued

\begin{tabular}{|c|c|c|c|c|c|c|c|c|c|c|c|c|c|}
\hline $\begin{array}{l}\text { Well } \\
\text { refer- } \\
\text { ence } \\
\text { number } \\
\text { (fig. 1) }\end{array}$ & Station number & $\begin{array}{c}\text { Sample } \\
\text { date }\end{array}$ & $\begin{array}{c}\text { Well } \\
\text { depth } \\
\text { (feet } \\
\text { below } \\
\text { LSD) }\end{array}$ & $\begin{array}{l}\text { Color, } \\
\text { (Pt-Co } \\
\text { units) }\end{array}$ & $\begin{array}{l}\mathrm{pH}, \\
\text { field }\end{array}$ & $\begin{array}{l}\mathrm{pH}, \\
\text { lab }\end{array}$ & $\begin{array}{c}\text { Specific } \\
\text { conduc- } \\
\text { tance, } \\
\text { lab } \\
(\mu \mathrm{S} / \mathrm{cm})\end{array}$ & $\begin{array}{c}\text { Specific } \\
\text { conduc- } \\
\text { tance, } \\
\text { field } \\
(\mu \mathrm{S} / \mathrm{cm})\end{array}$ & $\begin{array}{l}\text { Temper- } \\
\text { ature, } \\
\text { water } \\
(\operatorname{deg} \mathrm{C})\end{array}$ & $\begin{array}{l}\text { Chlor- } \\
\text { ide, } \\
\text { (mg/L) }\end{array}$ & $\begin{array}{l}\text { Total } \\
\text { dis- } \\
\text { solved } \\
\text { solids } \\
\text { (mg/L) }\end{array}$ & $\begin{array}{l}\text { Iron } \\
(\mu \mathrm{g} / \mathrm{L})\end{array}$ & $\begin{array}{c}\text { Mangan- } \\
\text { ese } \\
(\mu \mathrm{g} / \mathrm{L})\end{array}$ \\
\hline \multirow[t]{3}{*}{85} & 302342088515201 & $04 / 25 / 97$ & 740 & 20 & 9.4 & 9.2 & 450 & 441 & -- & 18 & 258 & -- & -- \\
\hline & 302342088515201 & 04/10/01 & 740 & 10 & 9 & 9.2 & 501 & 486 & 27 & 37 & 297 & 3 & 3 \\
\hline & 302342088515201 & 04/13/05 & 740 & 15 & 9.1 & 9.2 & 489 & 527 & 27.8 & 48 & 303 & E6 & 3.6 \\
\hline \multirow[t]{4}{*}{86} & 302441088523901 & $04 / 22 / 97$ & 1300 & 20 & 8.5 & 8.6 & 1530 & 1480 & 29 & 310 & 820 & 40 & 17 \\
\hline & 302441088523901 & 04/23/99 & 1300 & -- & 9.2 & 8.4 & 1560 & 1480 & 24 & 320 & -- & -- & -- \\
\hline & 302441088523901 & 04/12/01 & 1300 & 40 & 7.4 & 8.2 & 1590 & 1540 & 26 & 320 & 842 & 34 & 20 \\
\hline & 302441088523901 & $04 / 15 / 05$ & 1300 & -- & 8.1 & 8.2 & 1600 & 1660 & -- & 347 & 903 & 1650 & 47.7 \\
\hline \multirow[t]{2}{*}{87} & 302815088590301 & 04/09/98 & 751 & 10 & 9.3 & 9.1 & 295 & 295 & 26 & 5.4 & 194 & 30 & 4 \\
\hline & 302815088590301 & $04 / 17 / 02$ & 751 & 5 & 9.3 & 9.2 & 298 & 307 & -- & 5.5 & 191 & 8 & 3 \\
\hline 88 & 302638088555301 & $10 / 14 / 97$ & 1060 & 20 & 8.6 & 8.8 & 1030 & 1020 & 28.5 & 160 & 582 & 170 & 28 \\
\hline \multirow[t]{2}{*}{89} & 302611088572201 & $04 / 20 / 00$ & 900 & $<5$ & 7.7 & 9.1 & 328 & 325 & 27 & 7.9 & 200 & 3 & 7 \\
\hline & 302611088572201 & $04 / 29 / 04$ & 900 & 10 & 9 & 9 & 328 & 333 & 27 & 7.7 & 199 & $<2$ & 7 \\
\hline 90 & 305403088593401 & 05/03/04 & 950 & 9 & 9 & 9.1 & 353 & 366 & 28.5 & 14 & 215 & 5 & 5 \\
\hline \multirow[t]{2}{*}{91} & 301830089173701 & 04/19/00 & 966 & 5 & 8.8 & 8.8 & 525 & 529 & 27 & 32 & 318 & 9 & 7 \\
\hline & 301830089173701 & $04 / 27 / 04$ & 966 & 10 & 8.7 & 8.8 & 516 & 533 & 27.5 & 31 & 317 & 5 & 7 \\
\hline \multirow[t]{2}{*}{92} & 301832089171301 & 04/19/00 & 982 & 10 & 8.8 & 8.8 & 560 & 566 & 27.5 & 36 & 341 & 7 & 7 \\
\hline & 301832089171301 & $04 / 27 / 04$ & 982 & 10 & 9 & 8.9 & 552 & 585 & 24.5 & 36 & 340 & 42 & 12 \\
\hline \multirow[t]{3}{*}{93} & 301917089153101 & 04/23/97 & 858 & 10 & 8.9 & 8.9 & 642 & 574 & 27 & 48 & 376 & -- & -- \\
\hline & 301917089153101 & $04 / 12 / 01$ & 858 & 20 & 9 & 8.9 & 640 & 621 & 27 & 46 & 380 & $<2$ & 10 \\
\hline & 301917089153101 & $04 / 14 / 05$ & 858 & 30 & 8.6 & 8.9 & 594 & 626 & 27.4 & 47 & 369 & $<6$ & 11.5 \\
\hline \multirow[t]{2}{*}{94} & 302020089110001 & $04 / 19 / 00$ & 873 & 5 & 7.4 & 7.9 & 274 & 278 & 25.5 & 2.7 & 190 & 229 & 250 \\
\hline & 302020089110001 & $04 / 27 / 04$ & 873 & 10 & 7.4 & 8.1 & 254 & 288 & 25.5 & 2.9 & 194 & 112 & 242 \\
\hline 95 & 302139089084401 & $04 / 19 / 00$ & 862 & 5 & 7 & 8.4 & 245 & 247 & 26 & 3.1 & 175 & 11 & 42 \\
\hline \multicolumn{14}{|c|}{ Hattiesburg Aquifer } \\
\hline 96 & 303545089071002 & $04 / 18 / 02$ & 1140 & $<5$ & -- & 5.1 & 26 & 30 & -- & 4.9 & 20 & 3 & 3 \\
\hline \multirow[t]{3}{*}{97} & 303806089080301 & $04 / 23 / 97$ & 1412 & 10 & 8.8 & 8.7 & 254 & 246 & 30 & 2.1 & 156 & -- & -- \\
\hline & 303806089080301 & 04/09/01 & 1412 & $<5$ & 8.2 & 8.7 & 250 & 245 & 30 & 2.0 & 162 & 6 & 7 \\
\hline & 303806089080301 & $04 / 11 / 05$ & 1412 & 8 & 8.8 & 8.7 & 231 & 257 & -- & 1.9 & 164 & 6 & 7.5 \\
\hline \multirow[t]{2}{*}{98} & 303410089052801 & $10 / 30 / 96$ & 1414 & $<5$ & 9.2 & 9.2 & 463 & 458 & 30 & 5.1 & 286 & 9 & 3 \\
\hline & 303410089052801 & $04 / 15 / 02$ & 1414 & 5 & -- & 9.1 & 467 & 482 & 29.5 & 5.3 & 293 & 7 & 8 \\
\hline \multirow[t]{3}{*}{99} & 302308089183101 & $04 / 23 / 97$ & 1775 & 10 & 8.6 & 8.8 & 591 & 583 & 33 & 84 & 328 & -- & -- \\
\hline & 302308089183101 & 04/11/01 & 1775 & 5 & 8.8 & 8.8 & 593 & 584 & 32 & 84 & 341 & 6 & 22 \\
\hline & 302308089183101 & $04 / 14 / 05$ & 1775 & 12 & 8.6 & 8.6 & 591 & 635 & -- & 93 & 332 & 11 & 24.9 \\
\hline \multirow[t]{3}{*}{100} & 302812088592101 & $04 / 24 / 97$ & 1472 & 10 & 8.6 & 8.7 & 1270 & 1170 & -- & 230 & 704 & -- & -- \\
\hline & 302812088592101 & $04 / 12 / 01$ & 1472 & 20 & 8.8 & 8.6 & 1250 & 1200 & 23 & 220 & 700 & 6 & 24 \\
\hline & 302812088592101 & 04/13/05 & 1472 & 30 & 8.6 & 8.7 & 1220 & 1260 & -- & 219 & 679 & 9 & 23 \\
\hline
\end{tabular}


Appendix I. Water quality data for selected wells sampled from 1997 to 2005, Harrison County, Mississippi--Continued

\begin{tabular}{|c|c|c|c|c|c|c|c|c|c|c|c|c|c|}
\hline $\begin{array}{c}\text { Well } \\
\text { refer- } \\
\text { ence } \\
\text { number } \\
\text { (fig. 1) }\end{array}$ & Station number & $\begin{array}{c}\text { Sample } \\
\text { date }\end{array}$ & $\begin{array}{l}\text { Well } \\
\text { depth } \\
\text { (feet } \\
\text { below } \\
\text { LSD) }\end{array}$ & $\begin{array}{l}\text { Color, } \\
\text { (Pt-Co } \\
\text { units) }\end{array}$ & $\begin{array}{c}\mathrm{pH}, \\
\text { field }\end{array}$ & $\begin{array}{l}\text { pH, } \\
\text { lab }\end{array}$ & $\begin{array}{c}\text { Specific } \\
\text { conduc- } \\
\text { tance, } \\
\text { lab } \\
(\mu S / c m)\end{array}$ & $\begin{array}{c}\text { Specific } \\
\text { conduc- } \\
\text { tance, } \\
\text { field } \\
(\mu \mathrm{S} / \mathrm{cm})\end{array}$ & $\begin{array}{c}\text { Temper- } \\
\text { ature, } \\
\text { water } \\
\text { (deg C) }\end{array}$ & $\begin{array}{l}\text { Chlor- } \\
\text { ide, } \\
\text { (mg/L) }\end{array}$ & $\begin{array}{c}\text { Total } \\
\text { dis- } \\
\text { solved } \\
\text { solids } \\
\text { (mg/L) }\end{array}$ & $\begin{array}{c}\text { Iron } \\
\text { ( } \mu \mathrm{g} / \mathrm{L})\end{array}$ & $\begin{array}{c}\text { Mangan- } \\
\text { ese } \\
(\mu \mathrm{g} / \mathrm{L})\end{array}$ \\
\hline \multirow[t]{3}{*}{101} & 302204089102701 & $04 / 22 / 97$ & 1640 & 20 & 8.8 & 8.9 & 408 & 390 & -- & 25 & 236 & -- & -- \\
\hline & 302204089102701 & 04/10/01 & 1640 & 5 & 8.9 & 8.9 & 400 & 391 & 32 & 23 & 246 & 11 & 16 \\
\hline & 302204089102701 & $04 / 12 / 05$ & 1640 & 10 & 8.8 & 8.8 & 374 & 406 & -- & 24 & 234 & 18 & 18.2 \\
\hline \multirow[t]{2}{*}{102} & 302139089121001 & 04/07/98 & 1860 & 5 & -- & 8.7 & 631 & 623 & -- & 95 & 348 & 7 & 26 \\
\hline & 302139089121001 & $04 / 16 / 02$ & 1860 & $<5$ & 8.5 & 8.7 & 622 & 647 & -- & 93 & 356 & 4 & 24 \\
\hline \multirow[t]{3}{*}{103} & 302132089084901 & 04/07/98 & 1670 & 10 & 8.8 & 8.9 & 372 & 372 & 32 & 16 & 234 & 9 & 12 \\
\hline & 302132089084901 & 04/19/00 & 1670 & 5 & 8.7 & 8.8 & 371 & 380 & 29 & 16 & 230 & 6 & 11 \\
\hline & 302132089084901 & $04 / 27 / 04$ & 1670 & 10 & 8.8 & 8.7 & 390 & 406 & 31 & 21 & 234 & 3 & 12 \\
\hline \multicolumn{14}{|c|}{ Catahoula Aquifer } \\
\hline \multirow[t]{2}{*}{104} & 302447089165201 & $04 / 23 / 97$ & 2413 & 20 & 8.7 & 8.8 & 595 & 581 & -- & 76 & 328 & -- & -- \\
\hline & 302447089165201 & 04/11/01 & 2413 & 10 & 8.7 & 8.7 & 593 & 574 & 26 & 75 & 346 & 26 & 30 \\
\hline
\end{tabular}

\section{Appendix II. Quality assurance (OA) data for selected wells sampled in 2005, Harrison County, Mississippi}

[LSD, Land Surface Datum; Pt-Co, Platinum-Cobalt; uS/cm, Microsiemens per centimeter at 25 deg Celcius; mg/L, Milligrams per Liter; ug/L, Micrograms per Liter; --, indicates no data available; E, estimated; <, less than]

\begin{tabular}{|c|c|c|c|c|c|c|c|c|c|c|c|c|c|}
\hline $\begin{array}{l}\text { Well } \\
\text { refer- } \\
\text { ence } \\
\text { number } \\
\text { (fig. 1) }\end{array}$ & Station number & $\begin{array}{c}\text { OA } \\
\text { sample } \\
\text { type }\end{array}$ & $\begin{array}{l}\text { Sample } \\
\text { date }\end{array}$ & $\begin{array}{c}\text { Well } \\
\text { depth } \\
\text { (feet } \\
\text { below } \\
\text { LSD) }\end{array}$ & $\begin{array}{l}\mathrm{pH}, \\
\text { field }\end{array}$ & $\begin{array}{l}\mathrm{pH}, \\
\text { lab }\end{array}$ & $\begin{array}{c}\text { Specific } \\
\text { conduc- } \\
\text { tance, } \\
\text { lab } \\
(\mu \mathrm{S} / \mathrm{cm})\end{array}$ & $\begin{array}{c}\text { Specific } \\
\text { conduc- } \\
\text { tance, } \\
\text { field } \\
(\mu \mathrm{S} / \mathrm{cm})\end{array}$ & $\begin{array}{c}\text { Water } \\
\text { temper- } \\
\text { ature, } \\
(\operatorname{deg} C)\end{array}$ & $\begin{array}{c}\text { Chloride } \\
\text { (mg/L) }\end{array}$ & $\begin{array}{c}\text { Total } \\
\text { dissolved } \\
\text { solids } \\
\text { (mg/L) }\end{array}$ & $\begin{array}{l}\text { Iron } \\
(\mu \mathrm{g} / \mathrm{L})\end{array}$ & $\begin{array}{c}\text { Manganese } \\
(\mu \mathrm{g} / \mathrm{L})\end{array}$ \\
\hline 79 & 302304089020301 & Replicate & $04 / 12 / 05$ & 900 & -- & 8.6 & 259 & -- & -- & 3.51 & 182 & E5 & 8.4 \\
\hline 83 & 302701088593301 & $\begin{array}{c}\text { Field } \\
\text { equipment } \\
\text { blank }\end{array}$ & $04 / 13 / 05$ & 805 & -- & E7 & 3 & -- & -- & $<0.2$ & $<10$ & $<6$ & $<0.6$ \\
\hline 99 & 302308089183101 & Replicate & $04 / 14 / 05$ & 1775 & -- & 8.7 & 595 & -- & -- & 93.1 & 347 & 11 & 25.2 \\
\hline
\end{tabular}


6 Printed on recycled paper 